\title{
FINDING MINIMAL AND MAXIMAL SETS OF SPATIAL RELATIONSHIPS IN PICTORIAL RETRIEVAL SYSTEMS*
}

\author{
QING-LONG ZHANG ${ }^{\dagger}$ AND STEPHEN S.-T. YAU ${ }^{\ddagger}$
}

\begin{abstract}
Spatial reasoning is an important component in pictorial retrieval systems. There are two approaches to handling spatial relationships: the well-known one is to use algorithms on which most earlier work such as $[13,17,21]$ is based, and the recent one [30] is to construct deductive rules that allow spatial relationships to be deduced. Sistla et al. [30] developed a system of rules $\mathcal{R}$ on reasoning about basic spatial relationships that are of common interest in pictorial databases. In this paper, we consider the following two problems with that system of rules $\mathcal{R}$ : the deduction problem (that is, to deduce new spatial relationships from a given set $\boldsymbol{F}$ of spatial relationships) and the reduction problem (that is, to eliminate redundant spatial relationships from $\boldsymbol{F}$.

We use the mathematically simple matrix representation approach to show that these two problems can be solved by efficient (i.e., polynomial-time) algorithms. The time required by both of them is at most a constant multiple of the time to compute the transitive reduction of a directed graph with $n$ vertices or to compute the transitive closure of a directed graph with $n$ vertices or to perform $n \times n$ Boolean matrix multiplication, and thus is always bounded by time complexity $O\left(n^{3}\right)$ (and space complexity $O\left(n^{2}\right)$ ), where $n$ is the number of all involved objects.
\end{abstract}

1. Introduction. Image database systems have been much studied over the past 20 years. One of the most important problems in the design of image database systems is how images are stored in the image databases $[7,8,11,13,14,31]$. While the use of indexing to allow database accessing has been well established in traditional database systems, content-based picture indexing techniques need to be developed for facilitating pictorial information retrieval from a pictorial database.

Tanimoto [32] suggested the use of picture icons as picture indexes, thus introducing the concept of iconic indexing. Subsequently, Chang et al. [13] developed the concept of iconic indexing by introducing the $2 \mathrm{D}$ string representation of the image. The 2D string approach is based on the idea that the spatial knowledge contained in a real picture can be suitably represented by a symbolic picture (i.e., a matrix of symbols) where every symbol corresponds to a significant element of the image. The position of a symbol in the grid corresponds to the position of the centroid of the represented significant element. Depending on the application, the significant elements of the image can be pixels, lines, regions, and objects, etc. A 2D string representing

*The main results of this paper are from Chapter 2, the first author's Ph.D. Thesis, Department of Mathematics, Statistics, and Computer Science, University of Illinois at Chicago.

${ }^{\dagger}$ Member, IEEE, Control and Information Laboratory, Department of Mathematics, Statistics, and Computer Science, University of Illinois at Chicago, 322 Science and Engineering Offices, 851 South Morgan Street, Chicago, Illinois 60607, USA. E-mail: zhangq@math.uic.edu

${ }^{\ddagger}$ Fellow, IEEE, Control and Information Laboratory, Department of Mathematics, Statistics, and Computer Science, University of Illinois at Chicago, 322 Science and Engineering Offices, 851 South Morgan Street, Chicago, Illinois 60607, USA. E-mail: yau@uic.edu 
a symbolic picture is derived from the picture by orthogonally projecting its symbols by columns and by rows. This approach gives an efficient and natural way to construct iconic indexes for two-dimensional pictures. With the 2D string approach, the problem of pictorial information retrieval for $2 \mathrm{D}$ pictures becomes a problem of $2 \mathrm{D}$ string subsequence matching $[13,26]$. Since then, the 2D string approach has been studied further in the literature (see, e.g., $[4,15,33]\}$. Some forms of extensions of the $2 \mathrm{D}$ string approach can be found in $[9,10,12,24,25]$. For three-dimensional pictures, representations such as the octree [23, 27] were developed, and an extension of the $2 \mathrm{D}$ string to three dimensions was suggested in [14], and a unified approach to iconic indexing for 2D and 3D pictures was then proposed by Costagliola et al. [16]. Other methods on image representation and retrieval can be found in the literature (see, e.g., [6, 19, 21, 22, 29]).

Sistla et al. [30] developed a rule system $\mathcal{R}$ for reasoning about spatial relationships in picture retrieval systems. In their paper, a real picture is assumed to be associated with some meta-data describing its contents, that is, information about the objects in the picture, their properties, and the spatial or nonspatial relationships among them. This meta-data information is generated and stored in the database. Sistla et al. considered various spatial relationships: left-of, right-of, in-front-of, behind, above, below, inside, outside, and overlaps. For the first time, they presented a set of rules $\mathcal{R}$ that can be used to deduce new relationships from a given set of relationships. These rules are sound, and $\mathcal{R}$ is complete for 3D pictures. However, they presented a counterexample to show that $\mathcal{R}$ is incomplete for $2 \mathrm{D}$ pictures.

There are three obvious distinctions between the work of Sistla et al. [30] and the work such as $[13,17,21]$ on handling spatial relationships. First, the sets of spatial operators are not identical. For example, the operators overlaps, inside, and outside in [30] are not present in the other approaches. Second, the operators in [30] are defined by absolute spatial relationships among objects, while the operators in the other approaches are defined by relative spatial relationships among objects. For example, consider two significant objects $A$ and $B$ in a real picture. Then the spatial relationship " $A$ is left of B" (written as " $A$ left-of $B$ ") in [13] means that the position of the centroid of $A$ is left of that of $B$ (and we say " $A$ left-of $B$ " is relative), whereas in [30] it means that $A$ is absolutely left of $B$ (and we say " $A$ left-of $B$ " is absolute). Note that the operator left-of has the weaker meaning in [13] than in [30] in the sense that "A left-of $B$ " is true in [13] whenever it is true in [30], and "A left-of $B$ " is not necessarily true in [30] when it is true in [13]. Third, the approach to handling spatial relationships in [30] is to construct rules that allow spatial relationships to be deduced, but the other studies are based mostly on algorithms.

In this paper we consider the following two critically important problems with the system of rules $\mathcal{R}$ in general-purpose pictorial retrieval systems. The first one, called 
the deduction problem, is to deduce new spatial relationships from a given set $\boldsymbol{F}$ of spatial relationships. More precisely, we are interested in generating all deducible spatial relationships from $\boldsymbol{F}$ (i.e., the maximal set of $\boldsymbol{F}$ ). The second one, called the reduction problem, is to eliminate redundant spatial relationships from a given set $\boldsymbol{F}$ of spatial relationships. More precisely, we are interested in finding all nonredundant spatial relationships in $\boldsymbol{F}$ (i.e., the minimal set of $\boldsymbol{F}$ ). Suppose, for example, two spatial relationships in a real picture are specified: $A$ left-of $B$ and $B$ left-of $C$. Then the new spatial relationship $A$ left-of $C$ can be deduced from $A$ left-of $B$ and $B$ left-of $C$ by the deduction mechanism. Conversely, suppose three spatial relationships in a real picture are specified: $A$ left-of $B, B$ left-of $C$, and $A$ left-of $C$. Note that $A$ left-of $C$ is deducible from $A$ left-of $B$ and $B$ left-of $C$. Thus, $A$ left-of $C$ is redundant and can be deleted by the reduction mechanism. Both the deduction mechanism and reduction mechanism can be considered to be reverse procedures of each other, and should be invoked by the query-processing systems that retrieve images by content.

The deduction problem is crucial because of the following reasons [30]. First, current existing image-processing algorithms may not be able to detect all objects and spatial relationships in a picture. Although the missing information may have been introduced manually, some deducible relationships may have been left out in order to save time for entering these relationships manually. Thus, the content-based information about each picture, stored in the database, may not be complete. Second, the deducible spatial relationships may not have to be stored explicitly in the database in order to save storage space (also see the next paragraph).

The reduction problem is crucial because of the following reasons. First, for every picture stored in the database, the storage space requirement for the set of spatial relationships in the content-based information can be minimized by simply storing its minimal set. Second, the communication costs can be minimized by simply transmitting the minimal set for the set of spatial relationships in each picture if the content-based information needs to be transferred from one site to another site. Third, for a query specified by a user to find pictures satisfying certain spatial relationships among their objects, the execution time can be saved by simply retrieving pictures satisfying the minimal set of the set of these spatial relationships in the user query.

We use the mathematically simple matrix representation approach to show that, given a consistent set of spatial relationships $\boldsymbol{F}$, one can find its minimal and maximal sets from $\boldsymbol{F}$ under the system of rules $\mathcal{R}$ by efficient (i.e., polynomial-time) algorithms. The time required by both of them is at most a constant multiple of the time to compute the transitive reduction of a directed graph with $n$ vertices or to compute the transitive closure of a directed graph with $n$ vertices or to perform $n \times n$ Boolean matrix multiplication, and thus is always bounded by time complexity $O\left(n^{3}\right)$ (and space complexity $O\left(n^{2}\right)$ ), where $n$ is the number of all involved objects. We also show 
that the minimal set of $\boldsymbol{F}$ is unique.

The rest of this paper is organized as follows. In Section 2, we present the concepts, notations, definitions, and facts used in the remainder of the paper. In Section 3, we consider the deduction and reduction problems, and show how to find the maximal and minimal sets of a given consistent set of spatial relationships under $\mathcal{R}$. The main theorem is proved, and the detailed algorithms for finding minimal and maximal sets are also given in this section. Conclusions are presented in Section 4.

2. Definitions and Basic Facts. In this section we present some concepts, notations, definitions, and basic facts.

2.1. The Rules for Reasoning about Absolute Spatial Relationships. Here first recall the semantic definitions of absolute spatial relationships, introduced in $[30]$.

It is assumed that a three-dimensional picture $p$ consists of finitely many objects and each object in $p$ corresponds to a nonempty set of points in the three-dimensional Cartesian space (the left-handed coordinate system), where each point is given by its three $x$-, $y$ - and $z$-coordinates. Given an object $X$ in a picture $p, p(X)$ denotes its corresponding nonempty set of points. A two-dimensional picture is defined similarly. Let $p$ be a picture in which objects $A$ and $B$ are contained. Now define when $p$ satisfies the following absolute spatial relationships involving basic spatial relationship operators, left-of, right-of, above, below, behind, in-front-of, inside, outside, and overlaps.

- $p$ satisfies the relationship $A$ left-of $B$, stating that $A$ is to the left of $B$ in the picture $p$, iff the $x$-coordinate of each point in $p(A)$ is less than the $x$-coordinate of each point in $p(B)$.

- $p$ satisfies the relationship $A$ above $B$, stating that $A$ is above $B$ in the picture $p$, iff the $y$-coordinate of each point in $p(A)$ is greater than the $y$-coordinate of each point in $p(B)$.

- $p$ satisfies the relationship $A$ behind $B$, stating that $A$ is behind $B$ in the picture $p$, iff the $z$-coordinate of each point in $p(A)$ is greater than the $z$ coordinate of each point in $p(B)$.

- $p$ satisfies the relationship $A$ inside $B$, stating that $A$ is inside $B$ in the picture $p$, iff $p(A) \subseteq p(B)$.

- $p$ satisfies the relationship $A$ outside $B$, stating that $A$ is outside $B$ in the picture $p$, iff $p(A) \cap p(B)=\emptyset$.

- $p$ satisfies the relationship $A$ overlaps $B$, stating that $A$ overlaps $B$ in the picture $p$, iff $p(A) \cap p(B) \neq \emptyset$.

The semantics of spatial relationship symbols right-of, below, and in-front-of are defined similarly. Notice that these relationship symbols right-of, below, and in-front- 
of are actually duals of left-of, above, and behind, respectively.

A finite set of spatial relationships $\boldsymbol{F}$ is said to be consistent if there is a picture satisfying all the relationships in $\boldsymbol{F}$. A spatial relationship $r$ is said to be implied by a finite set of spatial relationships $\boldsymbol{F}$ if every picture satisfying all the relationships in $\boldsymbol{F}$ also satisfies the relationship $r$.

A deductive rule is in the following form

$$
r:: r_{1}, r_{2}, \ldots, r_{k}
$$

where $r$ and $r_{i}(1 \leq i \leq k, k \geq 0)$ are spatial relationships. The relationship $r$ and the list of relationships $r_{1}, r_{2}, \ldots, r_{k}$ are called the head and the body of the rule, respectively. A relationship $r$ is said to be deducible in one step from a set of relationships $\boldsymbol{F}$ by using a rule, if the head of the rule is $r$ and every relationship in the body of the rule is in $\boldsymbol{F}$. Let $\mathcal{R}$ be a set of rules. A relationship $r$ is said to be deducible from a set of relationships $\boldsymbol{F}$ by using the rules in $\mathcal{R}$, if $r$ is in $\boldsymbol{F}$, or there is a finite sequence of relationships $r_{1}, r_{2}, \ldots, r_{l}=r(l \geq 1)$, such that $r_{1}$ is deducible in one step from $\boldsymbol{F}$ by using a rule in $\mathcal{R}$, and for each $2 \leq i \leq l, r_{i}$ is deducible in one step from $\boldsymbol{F} \cup\left\{r_{1}, r_{2}, \ldots, r_{i-1}\right\}$ by using a rule in $\mathcal{R}$. The sequence $r_{1}, r_{2}, \ldots, r_{l}(=r)$ is called a derivation of $r$ from $\boldsymbol{F}$ by using the rules in $\mathcal{R}$ and $k$ is called the length of this derivation.

A deductive rule is called sound if every picture satisfying all the spatial relationships in the body of the rule also satisfies the spatial relationship given by the head of the rule. A set of rules $\mathcal{R}$ is called sound if every rule in $\mathcal{R}$ is sound. A set of rules $\mathcal{R}$ is said to be complete if it satisfies the following requirement for every consistent set of spatial relationships $\boldsymbol{F}$ : a spatial relationship implied by $\boldsymbol{F}$ is always deducible from $\boldsymbol{F}$ by using the rules in $\mathcal{R}$.

Now let us present the system of rules $\mathcal{R}$, rules I-VIII, introduced in [30], for reasoning about absolute spatial relationships.

I. (Transitivity of left-of, above, behind, and inside) For each $x \in\{$ left-of, above, behind, inside\}, we have

$A x C:: A x B, B x C$

II. For each $x \in\{$ left-of, above, behind $\}$, we have

$A x D:: A x B, B$ overlaps $C, C \times D$

III. For each $x \in\{$ left-of, above, behind, outside $\}$, we have the following two types of rules.

(a) $A x C::$ A inside $B, B x C$

(b) $A x C:: A x B, C$ inside $B$

IV. (Symmetry of overlaps and outside) For each $x \in\{$ overlaps, outside $\}$, we have $A x B:: B \times A$

V. For each $x \in\{$ left-of, above, behind $\}$, we have 
$A$ outside $B:: A x B$

VI. $A$ overlaps $B:: A$ inside $B$

VII. $A$ overlaps $B$ :: $C$ inside $A, C$ overlaps $B$

VIII. $A$ inside $A$ ::

For two-dimensional pictures, one does not have the spatial relationship symbol behind and the rules referring to it.

Notice that, the relationship symbols right-of, below, and in-front-of are excluded in the above rules of $\mathcal{R}$, since they are duals of left-of, above, and behind, respectively. They can be handled by additional rules that simply relate them to their duals (see rules IX-XI in [30]).

Sistla et al. [30] proved that the set of rules $\mathcal{R}$ given above is sound for twodimensional and three-dimensional pictures, and $\mathcal{R}$ is complete for three-dimensional pictures. However, they presented a counterexample to show that $\mathcal{R}$ is incomplete for two-dimensional connected pictures (Note that the connectedness requirement prevents an object in a picture from having disjoint parts). Without the connectedness assumption, $\mathcal{R}$ can also be shown to be complete for two-dimensional pictures.

Unless it is otherwise stated, $\mathcal{R}$ will be used to represent the set of rules I-VIII given above.

2.2. Minimal and Maximal Sets of Spatial Relationships. When we mention a set of spatial relationships $\boldsymbol{E}$ we always assume that $\boldsymbol{E}$ is consistent, that is, there exists a picture $p$ satisfying all the relationships in $\boldsymbol{E}$. Note that $p$ could have some objects that do not involve a relationship in $\boldsymbol{E}$. However, without loss of generality, we can assume that the maximal set of $\boldsymbol{E}$ defined below involves only those objects appearing in $\boldsymbol{E}$. Now we give the definitions of minimal and maximal sets.

Definition 2.1. Given a set $\boldsymbol{E}$ of spatial relationships, a subset $\boldsymbol{F} \subseteq \boldsymbol{E}$ is called a minimal set of $E$ under the system of rules $\mathcal{R}$ if (i) each $r \in \boldsymbol{E}$ is deducible from $\boldsymbol{F}$ using the rules in $\mathcal{R}$, and (ii) no proper subset of $\boldsymbol{F}$ satisfies condition (i).

Definition 2.2. Given a set $\boldsymbol{E}$ of spatial relationships, a superset $\boldsymbol{F} \supseteq \boldsymbol{E}$ is called a maximal set of $\boldsymbol{E}$ under the system of rules $\mathcal{R}$ if (i) each $r \in F$ is deducible from $\boldsymbol{E}$ using the rules in $\mathcal{R}$, and (ii) no proper superset of $\boldsymbol{F}$ satisfies condition (i).

Proposition 2.3 establishes the existence of the minimal sets.

Proposition 2.3. Given a set $\boldsymbol{E}$ of spatial relationships, there exists one minimal set $\boldsymbol{F}$ of $\boldsymbol{E}$ under $\mathcal{R}$.

Proof. Suppose $\boldsymbol{E}$ has $k$ relationships $r_{1}, r_{2}, \ldots, r_{k}$, that is, $\boldsymbol{E}=\left\{r_{1}, r_{2}, \ldots, r_{k}\right\}$. Now, if $r_{1}$ can be deducible from $\boldsymbol{E}-\left\{r_{1}\right\}$ using rules in $\mathcal{R}$, then $r_{1}$ is redundant and so can be deleted from $\boldsymbol{E}$; otherwise, $r_{1}$ cannot be deleted from $\boldsymbol{E}$. Thus, we define $\boldsymbol{E}_{1}$ to be $\boldsymbol{E}-\left\{r_{1}\right\}$ if $r_{1}$ is deducible from $\boldsymbol{E}-\left\{r_{1}\right\}$ by using rules in $\mathcal{R}$, and $\boldsymbol{E}$ otherwise. Similarly, we define $\boldsymbol{E}_{i}$ to be $\boldsymbol{E}_{i-1}-\left\{r_{i}\right\}$ if $r_{i}$ is deducible from $\boldsymbol{E}_{i-1}-\left\{r_{i}\right\}$ by using 
rules in $\mathcal{R}$, and $\boldsymbol{E}_{i-1}$ otherwise, where $2 \leq i \leq k$. Let $\boldsymbol{F}$ be $\boldsymbol{E}_{k}$. Then $\boldsymbol{F}$ is a minimal set of $\boldsymbol{E}$ under $\mathcal{R}$.

Proposition 2.4 establishes the existence and uniqueness of the maximal set.

Proposition 2.4. Given a set $\boldsymbol{E}$ of spatial relationships, there exists exactly one maximal set $\boldsymbol{F}$ of $\boldsymbol{E}$ under $\mathcal{R}$.

Proof. For each possible relationship $A x B$, where objects $A$ and $B$ appear in $\boldsymbol{E}$ and $x \in\{$ left-of, above, behind, inside, outside, overlaps $\}$, we put it into $\boldsymbol{F}$ if and only if it is deducible from $\boldsymbol{E}$ under $\mathcal{R}$. Then $\boldsymbol{F}$ satisfies the required properties.

The above two propositions only establish the existence of minimal and maximal sets. Later, we will show how to find them efficiently. We will also show that the minimal set is unique if we identify $A x B$ with $B x A$, where $A$ and $B$ are any involved objects and $x$ is either overlaps or outside.

2.3. Directed Graph, Transitive Closure, and Transitive Reduction. A directed graph (or digraph) $G$ is a subset of $V \times V$, where $V$ is a finite set. The elements in $V$ and $G$ are called the vertices and arcs of the graph, respectively. Given two vertices $u$ and $v$ in $V$, a directed path in $G$ from $u$ to $v$ is a sequence of distinct $\operatorname{arcs} \alpha_{1}, \alpha_{2}, \ldots, \alpha_{k}(k \geq 1)$, such that there exists a corresponding sequence of vertices $u=v_{0}, v_{1}, v_{2}, \ldots, v_{k}=v$ satisfying $\alpha_{i+1}=\left(v_{i}, v_{i+1}\right) \in G$, for $0 \leq i \leq k-1$. A cycle is a directed path beginning and ending at the same vertex and passing through at least one other vertex. An arc in the form $(v, v)$ is called a loop. A graph is called acyclic if it contains no cycles or loops.

A graph $G$ is called transitive if, for every pair of vertices $u$ and $v$, not necessarily distinct, $(u, v) \in G$ whenever there exists a directed path in $G$ from $u$ to $v$. The transitive closure $G^{T}$ of $G$ is the least subset of $V \times V$ that contains $G$ and is transitive.

Given a directed graph $G$, one often wishes to know whether there is a path from one vertex to another in $G$. This path information in the directed graph $G$ can possibly be represented by another directed graph with fewer arcs than $G$. In [28], a minimum equivalent graph of a directed graph $G$ is defined to be a smallest subgraph $G^{\prime}$ of $G$ which satisfies the following property for every pair of vertices $u$ and $v$ : there is a directed path from $u$ to $v$ in $G^{\prime}$ whenever there is a directed path from $u$ to $v$ in $G$. In [1], a transitive reduction of a directed graph $G$ is defined to be a graph $G^{t}$ which satisfies: (i) for every pair of vertices $u$ and $v$, there is a directed path from $u$ to $v$ in $G^{t}$ if and only if there is a directed path from $u$ to $v$ in $G$, and (ii) there is no graph that has fewer arcs than $G^{t}$ and meets condition (i). Notice that the important distinction between the notions of minimum equivalent graph and transitive reduction is that a transitive reduction should not be a subgraph of the original directed graph. Though the two notions yield the same unique reduced representation for a directed graph without cycles, the transitive reduction of a directed graph $G$ with cycles can 
be smaller and easier to compute than a minimum equivalent graph of $G$.

For a directed graph $G$ without cycles, the transitive reduction of $G$ can be found by successively checking the $\operatorname{arcs}$ of $G$ in any order and deleting those redundant arcs, where an $\operatorname{arc} \alpha=(u, v)$ is redundant if there is a directed path in $G$ from $u$ to $v$ which does not contain $\alpha$.

For a directed graph $G$ with cycles, the transitive reduction of $G$ may not be unique; that is, there may be more than one such graph, with fewest arcs, having the same transitive closure as $G$. However, in [1], all such minimal graphs for the given graph $G$ are showed to have similar structure, and a natural canonical representative can then be selected as the unique transitive reduction of $G$.

It was shown in [20] that finding the transitive closure of a graph with $n$ vertices requires at most a constant multiple of the time to perform $n \times n$ Boolean matrix multiplication, and the converse was shown in [18]. In their paper [1], Aho et al. provided an efficient algorithm for finding the transitive reduction of any given directed graph, and they showed that finding the transitive reduction of an $n$ vertex graph has the same time complexity as finding the transitive closure of an $n$ vertex graph or performing $n \times n$ Boolean matrix multiplication. Therefore, we have the following fact.

FACT 2.5. It takes the same equivalent time complexity to compute the transitive reduction of a graph with $n$ vertices, or to compute the transitive closure of a graph with $n$ vertices, or to perform $n \times n$ Boolean matrix multiplication.

In this paper, all directed graphs that we derive are acyclic. Hence, each of them has a unique transitive reduction.

Let $G$ be a directed graph. We will use $G^{T}$ and $G^{t}$, respectively, to denote the transitive closure and the transitive resduction of $G$. It is assumed that a directed graph $G$ is represented by its adjacency matrix $M$, the matrix with a 1 in row $i$ and column $j$ if there is an arc from the $i$ th vertex to the $j$ th vertex and a 0 there otherwise. For simplicity, sometimes we identify a graph $G$ with its adjacency matrix $M$, and also use $M^{T}$ and $M^{t}$, respectively, to denote adjacency matrices of the transitive closure $G^{T}$ and transitive reduction $G^{t}$. For a set $\boldsymbol{E}$ of " $x$ " relationships, where $x \in\{$ leftof, above, behind, inside, outside, overlaps\}, we also associate it with its adjacency matrix, the matrix with a 1 in row $i$ and column $j$ if the relationship "(the $i$ th object) $x$ (the $j$ th object)" is in $\boldsymbol{E}$ and a 0 there otherwise, and identify $\boldsymbol{E}$ with its adjacency matrix. However, the intended meaning will be clear from the context.

One important relationship between $G^{t}$ and $G^{T}$ of an acyclic graph $G$ is stated below. (It should be noted that the following Proposition 2.6 is indeed a straightforward consequence of Theorem 3 in [1].)

Proposition 2.6. $G^{t}=G-G * G^{T}$, where $G$ is an acyclic directed graph and multiplication ' $*$ ' denotes Boolean matrix multiplication (i.e., addition ' + ' and 
multiplication ' $*$ ' on two Boolean values are defined in this way: $0+0=0,0+1=1$, $1+0=1$, and $1+1=1 ; 0 * 0=0,0 * 1=0,1 * 0=0$, and $1 * 1=1)$.

Proof. Note that an $\operatorname{arc}(u, v)$ is in $G * G^{T}$ if and only if there exists some $w$, distinct from $u$ and $v$, such that $(u, w)$ is an $\operatorname{arc}$ of $G$ and there is a path from $w$ to $v$ in $G$. Now, an $\operatorname{arc}(u, v)$ is in $G^{t}$ if and only if $(u, v)$ is in $G$, and there does not exist any path from $u$ to $v$ in $G$ that does not contain arc $(u, v)$, in other words, if and only if $(u, v)$ is in $G$, but not in $G * G^{T}$.

Proposition 2.6 tells us that, for an acyclic directed graph $G$, the transitive reduction $G^{t}$ of $G$ can be easily computed from the transitive closure $G^{T}$ of $G$ by applying Boolean matrix multiplication (and subtraction). Notice that we can easily compute both $G^{T}$ and $G^{t}$ of an acyclic graph $G$ using efficient standard algorithms with time complexity $O\left(n^{3}\right)$ and space complexity $O\left(n^{2}\right)$. where $n$ is the total number of vertices in $G$ (see, e.g., $[1,2,3,5]$ ).

Let $\boldsymbol{S} \boldsymbol{R}$ be a set of spatial relationships and $\mathrm{n}$ be the number of all objects involved in $\boldsymbol{S R}$. We assume that these $n$ objects involved in $\boldsymbol{S} \boldsymbol{R}$ are always arranged in some order from first to $n$ th. Note that, two identical objects located in different positions in a real picture are represented by different subscripts among $1,2, \ldots, n$. This is required for the description of spatial relationships and the $2 \mathrm{D}$ string representation of a picture. Certainly they will be matched to the same object during pictorial retrieval.

DEFINITION 2.7. Let $\boldsymbol{S} \boldsymbol{R}$ be a set of spatial relationships and $x$ be a relationship symbol chosen from \{left-of, above, behind, inside\}. A dependency graph derived by $x$ (and $\boldsymbol{S} \boldsymbol{R}$ implicitly) is defined as a directed graph $G_{x}$, its vertex set is the set of all objects involved in $\boldsymbol{S} \boldsymbol{R}$, and an arc $(A, B)$ is in $G_{x}$ if and only if $A x B$ is in $\boldsymbol{S} \boldsymbol{R}$.

Note that, from Rule VIII, any relationship $A$ inside $A$ is always redundant for any involved object $A$ and thus could be deleted from $\boldsymbol{S} \boldsymbol{R}$ immediately when we begin to delete redundant relationships of $\boldsymbol{S} \boldsymbol{R}$. Furthermore, all of them must be added into the maximal set of $\boldsymbol{S} \boldsymbol{R}$ when we generate it. Therefore, we can assume that the derived dependency graph $G_{\text {inside }}$ does not include any $\operatorname{arc}(A, A)$. Now it is obvious that four derived dependency graphs, $G_{\text {left-of }}, G_{\text {above }}, G_{\text {behind }}$, and $G_{\text {inide }}$ are acyclic for any consistent set $\boldsymbol{S} \boldsymbol{R}$ of spatial relationships.

Let $\boldsymbol{E}$ be a set of spatial relationships and $x$ be a relationship symbol. We will use $\boldsymbol{E}^{x}$ to denote the subset of all " $x$ " relationships that are in $\boldsymbol{E}$. For example, if $\boldsymbol{E}=\{A$ left-of $B, B$ left-of $C, A$ outside $C\}$, then $\boldsymbol{E}^{\text {left-of }}=\{A$ left-of $B, B$ left-of $C\}$, $\boldsymbol{E}^{\text {outside }}=\{A$ outside $C\}$, and $\boldsymbol{E}^{\text {inside }}=\emptyset$. Let $\boldsymbol{F}$ be a set of spatial relationships involving only overlaps or outside. We will use $\boldsymbol{F}^{s}$ to denote the set of all corresponding symmetrical relationships from $\boldsymbol{F}$. For example, if $\boldsymbol{F}_{1}=\{A$ overlaps $B, C$ overlaps $D$, $D$ overlaps $C\}$, then $\boldsymbol{F}_{1}^{s}=\{B$ overlaps $A, D$ overlaps $C, C$ overlaps $D\}$, and if $\boldsymbol{F}_{2}=\{A$ outside B, C outside D $\}$, then $\boldsymbol{F}_{2}^{s}=\{B$ outside $A, D$ outside $C\}$. 
3. Deduction and Reduction Algorithms. Now we begin to present the deduction and reduction Algorithms. Note that, given a consistent set $\boldsymbol{S} \boldsymbol{R}$ of spatial relationships, deleting redundant relationships in $\boldsymbol{S} \boldsymbol{R}$ and deducing new relationships from $\boldsymbol{S R}$, under the system of rules $\mathcal{R}$, are both interleaving in some way.

We divide the deletion of redundant relationships in $\boldsymbol{S} \boldsymbol{R}$ under $\mathcal{R}$ into four parts: (i) deleting redundant relationships involving left-of, above, and behind; (ii) deleting redundant overlaps relationships; (iii) deleting redundant outside relationships; and (iv) deleting redundant inside relationships. Among these four parts, the first part is the hardest and the last part is the easiest. Generating all deducible relationships from $\boldsymbol{S R}$ under $\mathcal{R}$ will be done during the process of the above deletion.

We begin with Part (i).

3.1. Deleting left-of, above, and behind Relationships. We have only the first three rules, I, II, and III, to deduce relationships involving left-of, above, and behind. To apply Rule III to delete redundant relationships, we should guarantee that all deducible inside relationships be generated from $\boldsymbol{S R}$. To apply Rule II to delete redundant relationships, we should also guarantee that all deducible overlaps relationships be generated from $\boldsymbol{S R}$.

3.1.1. Generating inside Relationships. We have only rules I and VIII to deduce inside relationships. As mentioned before, $G_{\text {inside }}$ denotes the dependency graph derived by the relationship symbol inside (and $\boldsymbol{S R}$ ), which does not contain any $\operatorname{arc}(A, A)$, where $A$ is an object. Obviously the set of all deducible inside relationships is

$$
G_{\text {inside }}^{T} \cup\{A \text { inside } A \mid A \text { is any involved object }\},
$$

denoted by INSIDE. Later we will use the set

$\boldsymbol{I N S I D E}^{+}=G_{\text {inside }}^{T}=\boldsymbol{I N S I D E}-\{A$ inside $A \mid A$ is any involved object $\}$.

Suppose, for example, $\boldsymbol{S} \boldsymbol{R}^{\text {inside }}=\{A$ inside $B, B$ inside $C\}$. Then $\boldsymbol{I N S I D \boldsymbol { E } ^ { + } =}$ $\boldsymbol{S} \boldsymbol{R}^{\text {inside }} \cup\{A$ inside $C\}$.

3.1.2. Generating overlaps Relationships. We have only three rules, IV, VI, and VII, to deduce overlaps relationships.

Let $O_{0}=\boldsymbol{S} \boldsymbol{R}^{\text {overlaps }}, O_{1}=O_{0} \cup O_{0}^{s}$, and $O_{2}$ be the set of all deducible overlaps relationships from INSIDE using Rules VI and IV. $O_{1}$ and $O_{2}$ could have a nonempty intersection set. Note that $O_{1} \cup O_{2}$ is the set of all deducible overlaps relationships from $O_{0} \cup \boldsymbol{I N S I D E}$ using only Rules IV and VI.

When $C$ is set to be $A$, Rule VII will become

$$
A \text { overlaps } B:: A \text { inside } A, A \text { overlaps } B \text {, }
$$

and this is trivial by Rule VIII. Similarly, when $C$ is set to be $B$, Rule VII will become $A$ overlaps $B:: B$ inside $A, B$ overlaps $B$, 
and this is trivial by Rule IV, VI, and VIII. Thus, we can assume that $C$ is always not equal to $A$ or $B$ whenever we apply Rule VII.

Any new deducible relationships $A$ overlaps $B$ (i.e., not in $O_{1} \cup O_{2}$ ) should have to be obtained from $O_{1} \cup O_{2}$ and $\boldsymbol{I N S I D E ^ { + }}$ using Rule VII at least once and Rule IV. Let $O_{3}$ be the set of all overlaps relationships deducible in one step from $O_{1} \cup O_{2}$ and $\boldsymbol{I N S I D E ^ { + }}$ using Rule VII, and let $O_{4}=O_{3}^{s}$, and $O_{5}$ be the set of all overlaps relationships deducible in one step from $O_{4}$ and $\boldsymbol{I N S I D E ^ { + }}$ using Rule VII.

Suppose, for example, $\boldsymbol{S R}=\{C$ inside $A, D$ inside $B, C$ overlaps $D\}$. Then $O_{0}$ $=\{C$ overlaps $D\}, O_{1}=\{C$ overlaps $D, D$ overlaps $C\}, \boldsymbol{I N S I D E ^ { + }}=\{C$ inside $A$, $D$ inside $B\}$ and $O_{2}=\{C$ overlaps $A, A$ overlaps $C, D$ overlaps $B, B$ overlaps $D\} \cup\{z$ overlaps $z \mid z \in\{A, B, C, D\}\}$. All new deducible relationships in $O_{3}$ are $A$ overlaps $D$ and $B$ overlaps $C$, since

$$
\begin{aligned}
& A \text { overlaps } D:: C \text { inside } A, C \text { overlaps } D \\
& B \text { overlaps } C:: D \text { inside } B, D \text { overlaps } C \text {. }
\end{aligned}
$$

Hence, $O_{4}$ contains $D$ overlaps $A$ and $C$ overlaps $B$. Now all new deducible relationships in $O_{5}$ are $A$ overlaps $B$ and $B$ overlaps $A$, since

$$
\begin{aligned}
& A \text { overlaps } B:: C \text { inside } A, C \text { overlaps } B \\
& B \text { overlaps } A:: D \text { inside } B, D \text { overlaps } A .
\end{aligned}
$$

Claim 3.1. The set of all new (i.e., not in $O_{1} \cup O_{2}$ ) deducible overlaps relationships is contained in $O_{3} \cup O_{4} \cup O_{5}$. Therefore, the set of all deducible overlaps relationships is

$$
\bigcup_{i=1}^{5} O_{i}
$$

denoted by $\boldsymbol{O V E R L A P S}$.

Proof. The reader may refer to Appendix for the proof of Claim 3.1.

Note that, for each object $A, A$ inside $A$ is in INSIDE, so $A$ overlaps $A$ is in $\mathrm{O}_{2}$ by Rule VI, and thus is in $\boldsymbol{O V E R L A P S}$. Let

OVERLAPS ${ }^{+}=$OVERLAPS - $\{A$ overlaps $A \mid A$ is any involved object $\}$. We will use $\boldsymbol{O V E R L A P S ^ { + }}$ later.

3.1.3. Deletion of left-of, above, and behind Relationships. We now consider the deletion of redundant relationships involving left-of, above, and behind. This deletion process can be divided into three steps: (a) deleting those redundant relationships that can be deduced by using only Rule I; (b) deleting those redundant relationships that can be deduced by using only both Rules I and II; and (c) deleting those redundant relationships that can be deduced by using Rules I, II, and III. Since any redundant relationship involving left-of, above, and behind is deducible, in the 


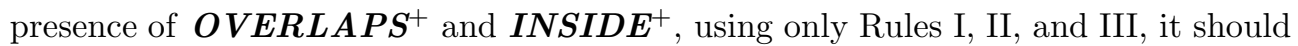
be removed from $\boldsymbol{S R}$ at one of the steps (a), (b), and (c).

Note that Rule II will become rule I whenever $B$ is identical to $C$, and Rule III will become trivial whenever $A$ is identical to $B$ for case (a) or $B$ is identical to $C$ for case (b). Hence, we can assume that applying Rule II requires the condition " $B$ is not identical to $C$ " and applying Rule III requires the condition " $A$ is not identical to $B$ for case (a) or $B$ is not identical to $C$ for case (b)."

The deletion process goes through (a), then (b), then (c), one time for each relationship symbol $x \in\{$ left-of, above, behind $\}$.

\section{Step (a) Using only Rule I}

Recall that $G_{x}$, defined in Section 2.3, is the dependency graph derived by $x$ (and $\boldsymbol{S R}$ implicitly) and is acyclic. It is obvious that, by Proposition 2.6, $G_{x}^{T}$ is the set of all " $x$ " relationships deducible by using only Rule I and $G_{x}^{t}=G_{x}-G_{x} * G_{x}^{T}$ is the set of left " $x$ " relationships that cannot be deleted by using only Rule I after this step.

Suppose, for example, $\boldsymbol{S R}=\{A$ above $B, B$ above $C, A$ above $C\}$. Then $G_{\text {above }}^{T}$ $=\boldsymbol{S} \boldsymbol{R}$ and $G_{\text {above }}^{t}=\{A$ above $B, B$ above $C\}$.

\section{Step (b) Using only Rules I and II}

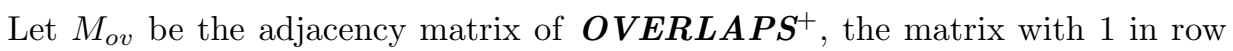
$i$ and column $j$ if the relationship "(the $i$ th object) overlaps (the $j$ th object)" is in $\boldsymbol{O V E R L A P S} S^{+}$and a 0 there otherwise. Then $G_{x}^{T} * M_{o v} * G_{x}^{T}$ represents the set of those " $x$ " relationships that are deducible in the presence of $\boldsymbol{O} \boldsymbol{V E R L A P \boldsymbol { S } ^ { + }}$ using Rule II exactly once and Rule I zero or more times. It is easy to see that $\bigcup_{2 \leq r \leq 3}\left(G_{x}^{T} * M_{o v}\right)^{r} * G_{x}^{T}$ represents the set of those " $x$ " relationships that are deducible in the presence of $\boldsymbol{O} \boldsymbol{V E R L A P \boldsymbol { S } ^ { + }}$ using Rule II exactly two times and Rule I zero or more times. Furthermore, $\bigcup_{r \geq 1}\left(G_{x}^{T} * M_{o v}\right)^{r} * G_{x}^{T}$ represents the set of those " $x$ " relationships that are deducible in the presence of $\boldsymbol{O} \boldsymbol{V E R} \boldsymbol{L} \boldsymbol{A P S ^ { + }}$, using Rule II at least once and Rule I zero or more times. Clearly $\bigcup_{r \geq 1}\left(G_{x}^{T} * M_{o v}\right)^{r} * G_{x}^{T}$ is the set of all new redundant " $x$ " relationships at this step, and thus should be removed from $G_{x}^{t}$. Therefore, the set of left " $x$ " relationships after step (b) is $G_{x}^{t}-\bigcup_{r \geq 1}\left(G_{x}^{T} * M_{o v}\right)^{r} * G_{x}^{T}$, denoted by $M_{x 1}$. And $\bigcup_{r \geq 0}\left(G_{x}^{T} * M_{o v}\right)^{r} * G_{x}^{T}$, denoted by $M_{x 2}$, is the set of all " $x$ "

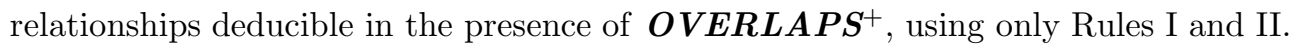

Let $M_{x}$ denote $G_{x}^{T} * M_{o v}$. It is easy to see that

$$
\begin{aligned}
M_{x 2} & =\bigcup_{r \geq 0}\left(G_{x}^{T} * M_{o v}\right)^{r} * G_{x}^{T} \\
& =G_{x}^{T}+\left(G_{x}^{T} * M_{o v}\right)^{T} * G_{x}^{T} \\
& =G_{x}^{T}+M_{x}^{T} * G_{x}^{T},
\end{aligned}
$$


and

$$
\begin{aligned}
M_{x 1} & =G_{x}^{t}-\bigcup_{r \geq 1}\left(G_{x}^{T} * M_{o v}\right)^{r} * G_{x}^{T} \\
& =G_{x}^{t}-\left(G_{x}^{T} * M_{o v}\right)^{T} * G_{x}^{T} \\
& =G_{x}^{t}-M_{x}^{T} * G_{x}^{T} .
\end{aligned}
$$

Note that $M_{x}=G_{x}^{T} * M_{o v}$ represents an "entire- $x$-partial" relation among objects, that is, $(A, C) \in G_{x}^{T} * M_{o v}$ if and only if there exists some object $B$ other than $A$ and $C$ such that $A x B$ and $B$ overlaps $C$, that means $A x(B \cap C)$, the entire object $A$ is $x$ to $B \cap C$, the part of the object $C$. This "entire- $x$-partial" relation among objects satisfies the transitive rule. Thus, $\bigcup_{r \geq 1}\left(G_{x}^{T} * M_{o v}\right)^{r}=\left(G_{x}^{T} * M_{o v}\right)^{T}=M_{x}^{T}$ is the transitive closure of $M_{x}=G_{x}^{T} * M_{o v}$. It is obvious that $M_{x 2}^{T}=M_{x 2}$.

Suppose, for example, $\boldsymbol{S R}=\{A$ above $B, C$ overlaps $B, C$ above $D, D$ above $E$, $C$ above $E, E$ overlaps $F, F$ above $G, A$ above $G\}$. At Step (a), $G_{\text {above }}^{T}=G_{\text {above }}=$ $\boldsymbol{S R}^{\text {above }}, G_{\text {above }} * G_{\text {above }}^{T}=\{C$ above $E\}$, and $G_{\text {above }}^{t}=\boldsymbol{S} \boldsymbol{R}^{\text {above }}-\{C$ above $E\}$. Hence,

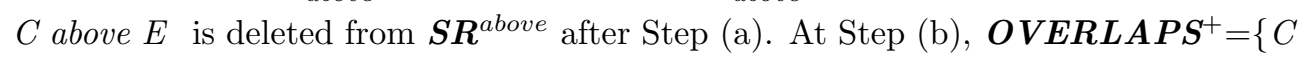
overlaps $B, B$ overlaps $C, E$ overlaps $F, F$ overlaps $E\}$. Then

$$
\begin{aligned}
& M_{\text {above }}=G_{\text {above }}^{T} * M_{o v}=\{(A, C),(D, F),(C, F)\}, \\
& M_{\text {above }}^{T}=M_{\text {above }} \cup\{(A, F)\}=\{(A, C),(D, F),(C, F),(A, F)\}
\end{aligned}
$$

and

$$
M_{\text {above }}^{T} * G_{\text {above }}^{T}=\{A \text { above } D, A \text { above } E, D \text { above } G, \text { Cabove } G, \text { Aabove } G\} .
$$

Since

$A$ above $D:: A$ above $B, B$ overlaps $C, C$ above $D$

$A$ above $E:: A$ above $E, B$ overlaps $C, C$ above $E$

$D$ above $G:: D$ above $E, E$ overlaps $F, F$ above $G$

$C$ above $G:: C$ above $E, E$ overlaps $F, F$ above $G$

$A$ above $G:: A$ above $D, D$ above $G$.

Therefore, $A$ above $G$ is deleted from $\boldsymbol{S R}^{\text {above }}$ after Step (b).

\section{Step (c) Using Rules I, II, and III}

For the purpose of ease of disposition, here we introduce the spatial relationship symbol contains, which says that $A$ contains $B$ iff $B$ inside $A$. Let

$\boldsymbol{C O N T A I N S}^{+}=\left\{A\right.$ contains $B \mid B$ inside $\left.A \in \boldsymbol{I N S I D E ^ { + }}\right\}$

and $M_{i n}$ and $M_{c o}$ respectively, be the adjacency matrices of the directed graphs $\boldsymbol{I N S I D E}^{+}$and $\boldsymbol{C O N T A I N S}^{+}$. Note that $\boldsymbol{I N S I D E ^ { + }}=G_{i n s i d e}^{T}$ and $M_{c o}=M_{i n}^{\prime}$, where $M_{i n}^{\prime}$ denotes the transpose matrix of $M_{i n}$.

After the spatial relationship symbol contains is introduced, Rule III(b) can be rewritten as follows: 
$A x C:: A x B, B$ contains $C$.

Now it is obvious that $M_{i n} * M_{x 2}$ and $M_{x 2} * M_{c o}$ (i.e., $M_{x 2} * M_{i n}^{\prime}$ ) represent the sets of all " $x$ " relationships deducible in one step from $M_{x 2}$ and $\boldsymbol{I N S I D \boldsymbol { E } ^ { + }}$ using Rule III(a) and Rule III(b), respectively. Furthermore, $M_{i n} * M_{x 2} * M_{c o}$ (i.e., $\left.M_{i n} * M_{x 2} * M_{i n}^{\prime}\right)$ represents the set of all " $x$ " relationships deducible in two steps

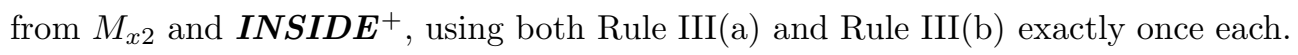
Furthermore, if $A x D \in M_{i n} M_{x 2} M_{c o}$, then

$$
A x D:: \quad A \text { inside } B, B x C, C \text { contains } D
$$

where

$$
A \text { inside } B \in \mathbf{I N S I D E ^ { + } , B} x C \in M_{x 2}
$$

and

$$
C \text { contains } D \in \text { CONTAINS }{ }^{+} \text {. }
$$

Note that $A x D$ can be derived by using Rule III(a) first, followed by using Rule III(b), that is,

$$
\begin{gathered}
A x C:: A \text { inside } B, B \times C \\
A x D: \because A x C, C \text { contains } D
\end{gathered}
$$

and $A x D$ can also be derived by using Rule III(b) first, followed by using Rule III(a), that is,

$$
\begin{gathered}
B \times D:: B \times C, C \text { contains } D \\
A x D:: A \text { inside } B, B \times D .
\end{gathered}
$$

Suppose, for example, $\boldsymbol{S R}=\{A$ inside $B, B$ above $C, D$ inside $C, A$ above $C, A$ above $D\}$. At Step (a), $G_{\text {above }}^{t}=G_{\text {above }}^{T}=G_{\text {above }}=\boldsymbol{S R}^{\text {above }}$. At Step (b), OVER$\boldsymbol{L} \boldsymbol{A} \boldsymbol{P} \boldsymbol{S}^{+}=\{A$ overlaps $B, B$ overlaps $A, D$ overlaps $C, C$ overlaps $D\}, M_{\text {above }}^{T}=$ $M_{\text {above }}=\{(A, D),(A, C),(B, D)\}$, and $M_{\text {above }}^{T} * G_{\text {above }}^{T}=\emptyset$. Hence, $M_{\text {above } 1}=M_{\text {above } 2}$ $=\boldsymbol{S} \boldsymbol{R}^{\text {above }}$. This means that no above relationships are deleted from $\boldsymbol{S} \boldsymbol{R}^{\text {above }}$ at Steps (a) and (b). At Step (c), INSIDE $\boldsymbol{E}^{+}=\{A$ inside $B, D$ inside $C\}$ and $\boldsymbol{C O N T A I N S}^{+}$ $=\{B$ contains $A, C$ contains $D\}$.

Then $M_{\text {in }} * M_{\text {above } 2}=\{A$ above $C\}$, since $A$ above $C:: A$ inside $B, B$ above $C$.

$M_{\text {above } 2} * M_{c o}=\{B$ above $D, A$ above $D\}$, since $B$ above $D:: B$ above $C, C$ contains $D$, $A$ above $D::$ A above $C, C$ contains $D$.

And $M_{\text {in }} * M_{\text {above } 2} * M_{c o}=\{A$ above $D\}$, since $A$ above $D:: A$ inside $B, B$ above $C, C$ contains $D$.

Claim 3.2. $M_{x 2} \cup M_{i n} M_{x 2} \cup M_{x 2} M_{i n}^{\prime} \cup M_{i n} M_{x 2} M_{i n}^{\prime}$, denoted by $\boldsymbol{M A X}(x)$, is the set of all " $x$ " relationships that are deducible, in the presence of $\boldsymbol{O V E R L A P \boldsymbol { S } ^ { + }}$

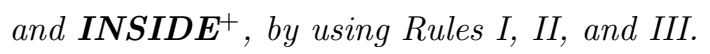

Proof. The reader may refer to Appendix for the proof of Claim 3.2. 
Therefore, $M_{i n} M_{x 2} \cup M_{x 2} M_{i n}^{\prime} \cup M_{i n} M_{x 2} M_{\text {in }}^{\prime}$ is the set of all new redundant " $x$ " relationships at Step (c) and should be removed from $M_{x 1}$. Then, the set of left " $x$ " relationships after Step (c) is

$$
M_{x 1}-\left(M_{i n} M_{x 2} \cup M_{x 2} M_{i n}^{\prime} \cup M_{i n} M_{x 2} M_{i n}^{\prime}\right),
$$

denoted by $\boldsymbol{M I N}(x)$. And it is obvious that $\boldsymbol{M A X}(x)^{T}=\boldsymbol{M A} \boldsymbol{X}(x)$.

3.2. Deleting overlaps Relationships. We already have $O_{0}, O_{1}, O_{2}, O_{3}, O_{4}$, and $O_{5}$ in the process of generating overlaps relationships in Section 3.1.2. Let $M_{12}$ be the adjacency matrix of $O_{1} \cup O_{2}$. Then it is easy to see that $O_{3}, O_{4}$, and $O_{5}$, respectively, have the adjacency matrices, $M_{i n}^{\prime} * M_{12},\left(M_{i n}^{\prime} * M_{12}\right)^{\prime}$ (i.e., $M_{12} * M_{i n}$, note that $M_{12}^{\prime}=M_{12}$ ), and $M_{i n}^{\prime} * M_{12} * M_{i n}$. Let $O=O_{0}-\bigcup_{i=2}^{5} O_{i}$, and $\boldsymbol{M I N}$ (overlaps) be one minimal set of $O$ under only the Symmetry Rule of overlaps. Note that MIN (overlaps) could have more than one choice from $O$. For example, if $O=$ $\{A$ overlaps $B, B$ overlaps $A$, Boverlaps $C\}$, then $M I N$ (overlaps) could be either $\{A$ overlaps $B, B$ overlaps $C\}$ or $\{B$ overlaps $A, B$ overlaps $C\}$. But if we identify $A$ overlaps $B$ with $B$ overlaps $A$ for any involved objects $A$ and $B$, then $\boldsymbol{M I N}$ (overlaps) is unique. Now $\boldsymbol{M I N}$ (overlaps) is just the minimal set of $\boldsymbol{S} \boldsymbol{R}^{\text {overlaps }}$ under the system of rules $\mathcal{R}$.

3.3. Generating and Deleting outside Relationships. We have only three rules, III(a) (Rule III(b) is redundant for the outside relationship), IV, and V, that can be used to deduce outside relationships. Because deducing outside relationships by using Rules III(a), IV, and V is similar to deducing overlaps relationships by using Rules VII, IV, and VI. Hence, we will generate all the outside relationships from $\boldsymbol{S} \boldsymbol{R}$ similar to generating all the overlaps relationships in Section 3.1.2. We also will use the outside deletion process similar to the overlaps deletion process in Section 3.2. We already have INSIDE from Section 3.1.1, and $\boldsymbol{M A X}(x)$ for each $x \in\{$ left-of, above, behind\} from Section 3.1.3.

Let $U_{0}=\boldsymbol{S} \boldsymbol{R}^{\text {outside }}, U_{1}=U_{0} \cup U_{0}^{s}$, and $U_{2}$ be the set of all deducible outside relationships from $\boldsymbol{M} \boldsymbol{A} \boldsymbol{X}$ (left-of) $\cup \boldsymbol{M} \boldsymbol{A} \boldsymbol{X}$ (above) $\cup \boldsymbol{M} \boldsymbol{A} \boldsymbol{X}$ (behind) by using Rules $\mathrm{V}$ and IV. Then $U_{1} \cup U_{2}$ is the set of all deducible outside relationships from $U_{0}$ and $\boldsymbol{M A X}(x)$, where $x \in\{$ left-of, above, behind $\}$, using only Rules IV and V.

Since, when $B$ is identical to $A$, Rule III(a) for " $x$ " chosen as "outside" will become

\section{$A$ outside $C: \because A$ inside $A, A$ outside $C$}

and this is trivial by Rule VIII. Thus, we can assume that $B$ is always not identical to $A$ whenever we apply Rule III(a) for outside relationships.

Any new deducible relationship $A$ outside $C$ (i.e., not in $U_{1} \cup U_{2}$ ) should have to be derived from $U_{1} \cup U_{2}$ and $\boldsymbol{I N S I D \boldsymbol { E } ^ { + }}$ using Rule III(a) at least once and Rule IV. Let $U 3$ be the set of all outside relationships deducible in one step from $U_{1} \cup U_{2}$ 
and $\boldsymbol{I N S I D E ^ { + }}$ using Rule III(a), and let $U_{4}=U_{3}^{s}$, and $U_{5}$ be the set of all outside relationships deducible in one step from $U_{4}$ and $\boldsymbol{I N S I D \boldsymbol { E } ^ { + }}$ using Rule III(a). Then, we have the following claim.

Claim 3.3. The set of all new (i.e., not in $U_{1} \cup U_{2}$ ) deducible outside relationships is contained in $U_{3} \cup U_{4} \cup U_{5}$. Therefore, the set of all deducible outside relationships is

$$
\bigcup_{i=1}^{5} U_{i}
$$

denoted by OUTSIDE.

Proof. Similar to the proof of Claim 3.1 in Section 3.1.2, which is placed in Appendix.

Let $U_{12}$ be the adjacency matrix of $U_{1} \cup U_{2}$. Then it is easy to see that $U_{3}, U_{4}$, and $U_{5}$, respectively, have the adjacency matrices $M_{i n} * U_{12},\left(M_{i n} * U_{12}\right)^{\prime}$ ( i. e., $U_{12} * M_{i n}^{\prime}$, Note that $\left.U_{12}=U_{12}^{\prime}\right)$, and $M_{i n} * U_{12} * M_{i n}^{\prime}$. Let $U=U_{0}-\cup_{i=2}^{5} U_{i}$, and MIN (outside) be one minimal set of $U$ under only the Symmetry Rule of outside. Note that $M I N$ (outside) could have more than one choice from $U$. For example, if $U=\{A$ outside $B, B$ outside $A, B$ outside $C\}$, then $\boldsymbol{M I N}$ (outside) could be either $\{A$ outside $B, B$ outside $C\}$ or $\{B$ outside $A, B$ outside $C\}$. But if we identify $A$ outside $B$ with $B$ outside $A$ for any involved objects $A$ and $B$, then $M I N$ (outside) is unique. Now $\boldsymbol{M I N}$ (outside) is just the minimal set of $\boldsymbol{S R}^{\text {outside }}$ under the system of rules $\mathcal{R}$.

3.4. Deleting inside Relationships. It is obvious that $G_{\text {inside }}^{t}$ is the minimal set of $\boldsymbol{S} \boldsymbol{R}^{\text {inside }}$ under the system of rules $\mathcal{R}$. This completes the deduction and reduction procedures.

3.5. The Minimal and Maximal Sets. It is easy to see that $\bigcup\{\boldsymbol{M I N}(x) \mid x \in\{$ left-of, above, behind, overlaps, outside $\}\} \bigcup G_{\text {inside }}^{t}$ is the minimal set of a given set $\boldsymbol{S R}$ of spatial relationships under the system of rules $\mathcal{R}$; and

$$
\bigcup\{\boldsymbol{M A X}(x) \mid x \in\{\text { left-of, above, behind }\}\} \bigcup \boldsymbol{O V E R L A P S} \bigcup \boldsymbol{O U T S I D E}
$$

UINSIDE

is the set of all spatial relationships deducible from $\boldsymbol{S R}$ using rules in $\mathcal{R}$, that is, the maximal set of $\boldsymbol{S} \boldsymbol{R}$ under $\mathcal{R}$. The minimal set of $\boldsymbol{S} \boldsymbol{R}$ is unique in the sense that we do not distinguish between $A x B$ and $B x A$ for any objects $A$ and $B$, and $x \in\{$ overlaps, outside\}.

The algorithms, for finding the minimal and maximal sets of a given consistent set of spatial relationships, are summarized as follows. In these algorithms, addition ' + ' and multiplication ' $*$ ' denote Boolean matrix addition and multiplication, 
respectively; subtraction '-' denotes Boolean matrix subtraction corresponding to the difference operation of two sets of " $x$ " relationships, where $x \in\{$ left-of, above, behind, inside, outside, overlaps $\}$, more precisely, let $X=\left(x_{i j}\right)_{n \times n}$ and $Y=\left(y_{i j}\right)_{n \times n}$ be two $n \times n$ Boolean matrices, then $X-Y$ is an $n \times n$ Boolean matrix $Z=\left(z_{i j}\right)_{n \times n}$ satisfying the condition that, for $1 \leq i, j \leq n, z_{i j}=x_{i j}-y_{i j}$, where the subtraction '-' on two Boolean values is defined in this way: $0-0=0,0-1=0,1-0=1$, and $1-1=0$. The following algorithms assume that we already have efficient standard algorithms for computing the transitive closure $G^{T}$ and transitive reduction $G^{t}$ of a given (acyclic) directed graph $G$. The algorithms for computing $G^{T}$ and $G^{t}$ of $G$ are represented by $\operatorname{TranC}\left(G, G^{T}\right)$ and $\operatorname{TranR}\left(G, G^{t}\right)$, respectively, where $G$ is a directed graph as input, and $G^{T}$ and $G^{t}$ are directed graphs as outputs of TranC and TranR, respectively. For each $x \in\{$ left-of, above, behind, inside, outside, overlaps $\}$, all sets of " $x$ " relationships are identified with their associated adjacency matrices. Let $I$ be an $n \times n$ identity matrix, where $n$ is the number of all objects involved in $\boldsymbol{S R}$. Then $I$ can denote either the set $\{A$ inside $A \mid A$ is any involved object $\}$ if the intended relationship is inside or the set $\{A$ overlaps $A \mid A$ is any involved object $\}$ if the intended relationship is overlaps.

Algorithm. Find the maximal set of a given consistent set of spatial relationships.

Input: a given consistent set $\boldsymbol{S R}$ of spatial relationships.

Output: the maximal set of $\boldsymbol{S R}$.

Step (1). Generate inside relationships

/* $G_{\text {inside }}$ denotes the dependency graph derived by inside and $\boldsymbol{S R} * /$

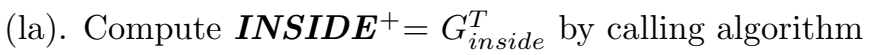

$\operatorname{TranC}\left(G_{\text {inside }}, \boldsymbol{I N S I D E ^ { + }}\right)$;

(lb). INSIDE $=\boldsymbol{I N S I D E ^ { + } + I .}$

Step (2). Generate overlaps relationships

/* $O_{0}=\boldsymbol{S} \boldsymbol{R}^{\text {overlaps }}$ denotes the subset of all overlaps relationships in $\boldsymbol{S R} * /$

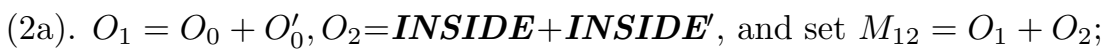

${ }^{*} M_{i n}$ is the adjacency matrix of $\boldsymbol{I N S I D E ^ { + * }}{ }^{*}$

(2b). $O_{3}=M_{i n}^{\prime} * M_{12}, O_{4}=O_{3}^{\prime}$ and $O_{5}=O_{3} * M_{i n}$;

(2c). OVERLAPS $=M_{12}+O_{3}+O_{4}+O_{5}$, and set

OVERLAPS ${ }^{+}=$OVERLAPS $-I$.

Step (3). Generate left-of, above, and behind relationships

$/ * G_{x}$ denotes the dependency graph derived by $x$ and $\boldsymbol{S R} * /$

For each $x \in\{$ left-of, above, behind $\}$, go through (3a)-(3c):

(3a). Compute $G_{x}^{T}$ by calling algorithm $\operatorname{TranC}\left(G_{x}, G_{x}^{T}\right)$;

/* $M_{o v}$ is the adjacency matrix of $\boldsymbol{O V E R L A P \boldsymbol { S } ^ { + * }}$ /

(3b). $M_{x}=G_{x}^{T} * M_{o v}$, and compute $M_{x}^{T}$ by calling algorithm 
$\operatorname{TranC}\left(M_{x}, M_{x}^{T}\right)$

then set $M_{x 2}=G_{x}^{T}+M_{x}^{T} * G_{x}^{T}$;

(3c). $\boldsymbol{M} \boldsymbol{A} \boldsymbol{X}(x)=M_{x 2}+M_{i n} * M_{x 2}+M_{x 2} * M_{i n}^{\prime}+M_{i n} * M_{x 2} * M_{i n}^{\prime}$.

Step (4). Generate outside relationships

$/ * U_{0}=\boldsymbol{S} \boldsymbol{R}^{\text {outside }}$ denotes the subset of all outside relationships in $\boldsymbol{S R} * /$

(4a). $U_{1}=U_{0}+U_{0}^{\prime}, U_{2}=\boldsymbol{M} \boldsymbol{A} \boldsymbol{X}$ (left-of) $+\boldsymbol{M A} \boldsymbol{X}$ (above) $+\boldsymbol{M A} \boldsymbol{X}$ (behind)

and reset $U_{2}=U_{2}+U_{2}^{\prime}$, then $U_{12}=U_{1}+U_{2}$;

(4b). $U_{3}=M_{i n} * U_{12}, U_{4}=U_{3}^{\prime}$ and $U_{5}=M_{i n} * U_{4}$;

(4c). OUTSIDE $=U_{12}+U_{3}+U_{4}+U_{5}$.

$/ *$ End of the algorithm for finding the maximal set $* /$

Algorithm. Find the minimal set of a given consistent set of spatial relationships.

Input: a given consistent set $\boldsymbol{S} \boldsymbol{R}$ of spatial relationships.

Output: the minimal set of $\boldsymbol{S} \boldsymbol{R}$.

Step (1). Delete left-of, above, and behind relationships

(la). Generate inside relationships

Same as Step (1) in the algorithm for finding the maximal set.

(lb). Generate overlaps relationships

Same as Step (2) in the algorithm for finding the maximal set.

(lc). Main deletion process

/* $G_{x}$ denotes the dependency graph derived by $x$ and $\boldsymbol{S} \boldsymbol{R} *$ /

For each $x \in\{$ left-of, above, behind $\}$, go through (lc.l)-(lc.3):

(lc.l). Same as (3a) in the algorithm for finding the maximal set.

(lc.2). Same as (3b) in the algorithm for finding the maximal set.

(lc.3). Compute $G_{x}^{t}$ by calling algorithm $\operatorname{TranR}\left(G_{x}, G_{x}^{t}\right)$,

then set $M_{x 1}=G_{x}^{t}-M_{x}^{T} * G_{x}^{T}$ and

$\operatorname{MIN}(x)=M_{x l}-\left(M_{i n} * M_{x 2}+M_{x 2} * M_{i n}^{\prime}+M_{i n} * M_{x 2} * M_{i n}^{\prime}\right)$.

Step (2). Delete overlaps relationships

(2a). Same as (2a) in the algorithm for finding the maximal set.

(2b). Same as (2b) in the algorithm for finding the maximal set.

(2c). $O=O_{0}-\left(O_{2}+O_{3}+O_{4}+O_{5}\right)$, and set $M I N$ (overlaps)

be one minimal set of $O$ under only the Symmetry Rule of overlaps.

Step (3). Delete outside relationships

(3a). Same as (4a) in the algorithm for finding the maximal set.

(3b). Same as (4b) in the algorithm for finding the maximal set.

(3c). $U=U_{0}-\left(U_{2}+U_{3}+U_{4}+U_{5}\right)$, and set $\boldsymbol{M I N}$ (outside)

be one minimal set of $U$ under only the Symmetry Rule of outside.

Step (4). Delete inside relationships 
/* $G_{\text {inside }}$ denotes the dependency graph derived by inside and $\boldsymbol{S} \boldsymbol{R} * /$

(4a). Compute $G_{\text {inside }}^{t}$ by calling algorithm $\operatorname{TranR}\left(G_{\text {inside }}, G_{\text {inside }}^{t}\right)$.

/* End of the algorithm for finding the minimal set */

At Step (2c) of the algorithm for finding the minimal set, MIN(overlaps) can be obtained from $O$ as follows. Let $W=\left(w_{i j}\right)_{n \times n}$ be an $n \times n$ matrix with all zero entries initially, where $n$ is the number of objects involved in $\boldsymbol{S R}$. For each relationship "the $i$ th object overlaps the $j$ th object" (denoted by $r$ ) in $O$, check " $w_{i j}=1$ ?". If $w_{i j}=1$, then continue to test the next available relationship in $O$; otherwise, put $r$ in $\boldsymbol{M I N}$ (overlaps) and set $w_{i j}=w_{j i}=1$, then continue to test the next available relationship in $O$. Clearly, each relationship in $O$ is tested only once. Thus, MIN (overlaps) can be found from $O$ in time $O\left(n^{2}\right)$, since the number of relationships in $O$ is bounded by $n^{2}$. Similarly, $\boldsymbol{M I N}$ (outside) can also be found from $U$ in time $O\left(n^{2}\right)$ at Step (3c).

It is easy to see that, for the above two algorithms, every computation at each step, excluding computing the transitive reduction and transitive closure of a directed graph and performing Boolean matrix multiplication, can be done by time complexity $O\left(n^{2}\right)$ and space complexity $O\left(n^{2}\right)$. Notice that computing the transitive reduction of a directed graph or computing the transitive closure of a directed graph or performing Boolean matrix multiplication each has to take at least time $O\left(n^{2}\right)$. For example, as mentioned in [1], an algorithm, for finding the transitive reduction in any reasonable graph representation, will have to examine all the arcs and thus require at least time $O\left(n^{2}\right)$. Hence, by Fact 2.5, each of both the above algorithms will require time that is at most a constant multiple of the time to compute the transitive reduction of a graph or to compute the transitive closure of a graph or to perform Boolean matrix multiplication. Note that we can easily compute both $G^{T}$ and $G^{t}$ of an acyclic graph $G$, using efficient standard algorithms with time complexity $O\left(n^{3}\right)$ and space complexity $O\left(n^{2}\right)$ (see, e.g., $[1,2,3,5]$ ), and perform Boolean matrix multiplication using usual matrix multiplication with time complexity $O\left(n^{3}\right)$ and space complexity $O\left(n^{2}\right)$. Therefore, the time complexity and space complexity of both the above algorithms are bounded by $O\left(n^{3}\right)$ and $O\left(n^{2}\right)$, respectively. Now we have the following theorem.

TheOREm 3.4. Given a consistent set of spatial relationships $\boldsymbol{S R}$, one can find its minimal and maximal sets from $\boldsymbol{S} \boldsymbol{R}$ under the system of rules $\mathcal{R}$ by efficient algorithms. The time required by both of them is at most a constant multiple of the time to compute the transitive reduction of a directed graph with $n$ vertices or to compute the transitive closure of a directed graph with $n$ vertices or to perform $n \times n$ Boolean matrix multiplication, and thus is always bounded by time complexity $O\left(n^{3}\right)$ (and space complexity $O\left(n^{2}\right)$ ), where $n$ is the number of all involved objects. The minimal set of $\boldsymbol{S R}$ is unique in the sense that one does not distinguish between AxB 
and $B x A$ for any objects $A$ and $B$, and $x \in\{$ overlaps, outside $\}$.

Given a consistent set $\boldsymbol{S R}$ of three-dimensional spatial relationships, we can use the above two algorithms to find the minimal and maximal sets of $S R$ under the system of rules $\mathcal{R}$. Since $\mathcal{R}$ is complete for three-dimensional pictures, the minimal and maximal sets of $\boldsymbol{S} \boldsymbol{R}$ under $\mathcal{R}$ coincide with the minimal and maximal sets implied by $\boldsymbol{S R}$ under $\mathcal{R}$, respectively. For two-dimensional pictures, we will not have the relationship symbol behind and the rules referring to it in $\mathcal{R}$. Similarly, we can use the above two algorithms (discarding those computations involving behind relationships) to find the minimal and maximal sets of a given consistent set $\boldsymbol{F}$ of two-dimensional spatial relationships under $\mathcal{R}$. However, since $\mathcal{R}$ is incomplete for two-dimensional connected pictures, the minimal and maximal sets of $\boldsymbol{F}$ under $\mathcal{R}$ may not coincide with the minimal and maximal sets implied by $\boldsymbol{F}$ under $\mathcal{R}$, respectively. More precisely, the minimal set of $\boldsymbol{F}$ under $\mathcal{R}$ may contain properly the minimal set implied by $\boldsymbol{F}$ under $\mathcal{R}$, while the maximal set of $\boldsymbol{F}$ under $\mathcal{R}$ may be contained properly in the maximal set implied by $\boldsymbol{F}$ under $\mathcal{R}$.

4. Conclusions. In this paper we have studied the deduction and reduction problems. We have shown that, given a consistent set of spatial relationships $\boldsymbol{S R}$, one can find its minimal and maximal sets from $\boldsymbol{S R}$ under the system of rules $\mathcal{R}$ by efficient (i.e., polynomial-time) algorithms. The time required by both of them is at most a constant multiple of the time to compute the transitive reduction of a directed graph with $n$ vertices or to compute the transitive closure of a directed graph with $n$ vertices or to perform $n \times n$ Boolean matrix multiplication, and thus is always bounded by time complexity $O\left(n^{3}\right)$ (and space complexity $O\left(n^{2}\right)$ ), where $n$ is the number of all involved objects. The minimal set of $\boldsymbol{S R}$ is unique in the sense that one does not distinguish between $A x B$ and $B x A$ for any objects $A$ and $B$, and $x \in\{$ overlaps, outside $\}$. The detailed algorithms are also given and can be directly programmed into executable computer codes.

We note that both the deduction mechanism and reduction mechanism can be implemented by an existing deductive system. However, the performance is likely to be much less efficient than the algorithmic approach we have presented in this paper.

The interested reader may refer to $[34,35,36,37.39,41,42,43,44]$ for our further developments in content-based image database systems. In particular, in our paper [39], we proposed the consistency problem on content-based pictorial description in pictorial database systems. We then suggested a framework for Content-based Image Database Systems (CIDBS). In [38], We also considered the consistency problem for spatial relationships in a picture, and used the same approach of mathematically simple matrix representation as in this paper to present an efficient (i.e., polynomialtime) algorithm for consistency checking of spatial relationships. Future research is 
required to further investigate the CIDBS model for facilitating fast image indexing and retrieval.

Appendix. In this appendix, we provide the complete proofs of Claim 3.1 in Section 3.1.2 and Claim 3.2 in Section 3.1.3.

Proof. (Proof of Claim 3.1)

Let $A$ overlaps $B$ be any new deducible relationship. Hence, $A$ overlaps $B$ is not in $U_{1} \cup U_{2}$. Let $k$ be the minimum length of a derivation of $A$ overlaps $B$ from $O_{1} \cup O_{2}$ and $\boldsymbol{I N S I D \boldsymbol { E } ^ { + }}$ using Rules VII and IV. We use induction on $k$ to prove that $A$ overlaps $B$ is in $O_{3} \cup O_{4} \cup O_{5}$.

$k=1$ : Then $A$ overlaps $B$ should be deducible in one step from $O_{1} \cup O_{2}$ and INSIDE ${ }^{+}$ using Rule VII. Hence, $A$ overlaps $B$ is in $O_{3}$.

$k=2$ :

If the last step in the derivation uses Rule VII, then

$$
\begin{aligned}
A \text { overlaps } B & :: C \text { inside } A, C \text { overlaps } B \\
\text { where } & C \text { inside } A \in \mathbf{I N S I D E ^ { + }} \\
\text { and } & C \text { overlaps } B \text { is still new. }
\end{aligned}
$$

But $C$ overlaps $B$ now is deducible in one step from $O_{1} \cup O_{2}$ and $\boldsymbol{I N S I D E ^ { + }}$ using Rule VII:

$$
\begin{aligned}
C \text { overlaps } B & :: D \text { inside } C, D \text { overlaps } B \\
\text { where } & D \text { inside } C \in \boldsymbol{I N S I D E}^{+} \\
\text {and } & D \text { overlaps } B \in O_{1} \cup O_{2} .
\end{aligned}
$$

Combining these two steps into one step, we have

$$
\begin{aligned}
\text { A overlaps } B & :: D \text { inside } A, D \text { overlaps } B \\
\text { where } & D \text { inside } A \in \boldsymbol{I N S I D \boldsymbol { E } ^ { + }} \\
\text { and } & D \text { overlaps } B \in O_{1} \cup O_{2} .
\end{aligned}
$$

Hence, $k=1$, contradicting the assumption $k=2$. Thus, the last step in the derivation cannot use Rule VII.

If the last step in the derivation uses Rule IV, then

$$
\begin{aligned}
& \text { A overlaps } B:: B \text { overlaps } A \\
& \text { where } B \text { overlaps } A \text { is still new. }
\end{aligned}
$$

Clearly $B$ overlaps $A$ now is deducible in one step from $O_{1} \cup O_{2}$ and $\boldsymbol{I N S I D E ^ { + }}$ using Rule VII. Hence, $B$ overlaps $A$ is in $O_{3}$, and thus $A$ overlaps $B$ is in $O_{4}$. 
$k=3:$

If the last step in the derivation uses Rule VII, then (1) holds. Now the minimum length of a derivation of $C$ overlaps $B$ from $O_{1} \cup O_{2}$ and $\boldsymbol{I N S I D \boldsymbol { E } ^ { + }}$ using Rules VII and IV is 2. Hence, $C$ overlaps $B$ is in $O_{4}$ from the case $k=2$, and thus $A$ overlaps $B$ is in $O_{5}$.

If the last step in the derivation uses Rule IV, then (2) holds. Clearly $B$ overlaps $A$ now is deducible in two steps from $O_{1} \cup O_{2}$ and $\boldsymbol{I N S I D E ^ { + }}$ using Rules VII and IV. Hence, $B$ overlaps $A$ is in $O_{4}$ from the case $k=2$, and thus $A$ overlaps $B$ should be in $O_{3}$. Therefore, $k=1$, contradicting the assumption $k=3$. This means that the last step in the derivation cannot use rule IV.

$k=4$ :

If the last step in the derivation uses Rule VII, then (1) holds. Now the minimum length of a derivation of $C$ overlaps $B$ from $O_{1} \cup O_{2}$ and $\boldsymbol{I N S I D E ^ { + }}$ using Rules VII and IV is 3 . Hence, $C$ overlaps $B$ is in $O_{5}$ from the case $k=3$. Thus, we have

$$
\begin{aligned}
C \text { overlaps } B & :: D \text { inside } C, D \text { overlaps } B \\
\text { where } & D \text { inside } C \in \mathbf{I N S I D E}^{+} \\
\text {and } & D \text { overlaps } B \in O_{4} .
\end{aligned}
$$

Combining both derivations of $A$ overlaps $B$ and $C$ overlaps $B$, we have

$$
\begin{gathered}
\text { A overlaps } B:: D \text { inside } A, D \text { overlaps } B \\
\text { where } \quad D \text { inside } A \in \boldsymbol{I N S I D E}^{+} \\
\text {and } \quad D \text { overlaps } B \in O_{4} .
\end{gathered}
$$

Therefore, $A$ overlaps $B$ has the minimum length 3 of a derivation from $O_{1} \cup O_{2}$ and INSIDE $\boldsymbol{E}^{+}$using Rules VII and IV, contradicting the assumption $k=4$. Thus, the last step in the derivation cannot use Rule VII.

If the last step in the derivation uses Rule IV, then (2) holds. Clearly $B$ overlaps $A$ now is deducible in three steps from $O_{1} \cup O_{2}$ and $\boldsymbol{I N S I D E ^ { + }}$ using Rules VII and IV. Hence, $B$ overlaps $A$ is in $O_{5}$ from the case $k=3$. Thus we have

$$
\begin{gathered}
B \text { overlaps } A:: C \text { inside } B, C \text { overlaps } A \\
\text { where } \quad C \text { inside } B \in \mathbf{I N S I D E}^{+} \\
\text {and } \quad C \text { overlaps } A \in O_{4} .
\end{gathered}
$$

Since $C$ overlaps $A$ is in $O_{4}$, it can be derived from $A$ overlaps $C$ using Rule IV, where $A$ overlaps $C \in O_{3}$. Then

$$
\begin{aligned}
\text { A overlaps } C & :: D \text { inside } A, D \text { overlaps } C \\
\text { where } & D \text { inside } A \in \mathbf{I N S I D E}^{+} \\
\text {and } & D \text { overlaps } C \in O_{1} \cup O_{2} .
\end{aligned}
$$


Now we have the following derivation of $A$ overlaps $B$ from $O_{1} \cup O_{2}$ and $\boldsymbol{I N S I D E}^{+}$ using Rules VII and IV (note that both $C$ overlaps $D$ and $D$ overlaps $C$ are in $O_{1} \cup O_{2}$ at the same time):

$$
\begin{gathered}
B \text { overlaps } D:: C \text { inside } B, C \text { overlaps } D \\
D \text { overlaps } B:: \quad B \text { overlaps } D \\
A \text { overlaps } B:: D \text { inside } A, D \text { overlaps } B
\end{gathered}
$$

Therefore, $k \leq 3$, contradicting the assumption $k=4$. Thus, the last step in the derivation also cannot use Rule IV. Therefore, $k$ cannot be 4 .

$k \geq 5$ :

We use induction on $k$ to prove that this case is impossible. Recall that $k$ cannot be 4 . Assume that there is a situation such that $k \geq 5$. Then, the last step in the derivation of $A$ overlaps $B$ uses either Rule VII or Rule IV. If the former rule is used, then (1) holds. Clearly $C$ overlaps $B$ now has the minimum length $(k-1) \geq 4$ of a derivation from $O_{1} \cup O_{2}$ and $\boldsymbol{I N S I D E ^ { + }}$ using Rules VII and IV, and by inductive assumption, this is impossible. If the latter rule is used, then (2) holds. Clearly $B$ overlaps $A$ now has the minimum length $\left(k-1 \geq 4\right.$ of a derivation from $O_{1} \cup O_{2}$ and $\boldsymbol{I N S I D E}^{+}$ using Rules VII and IV, and by inductive assumption, this is also impossible.

Thus, we have now proved that $k$ is at most 3 . This means that any new deducible overlaps relationships (i.e., not in $O_{1} \cup O_{2}$ ) can be derived within three steps from $O_{1} \cup O_{2}$ and $\boldsymbol{I N S I D E ^ { + }}$ using Rules VII and IV.

This completes the proof of Claim 3.1.

Proof. (Proof of Claim 3.2)

Recall that $M_{x 2}$ is the set of all " $x$ " spatial relationships deducible, in the presence of $O V E R L A P S^{+}$(and INSIDE ${ }^{+}$), by using only Rules I and II. Let $P_{k}$ be the set of all " $x$ " spatial relationships deducible within $k$ steps, in the presence of $\boldsymbol{O V E R L A P \boldsymbol { S } ^ { + }}$

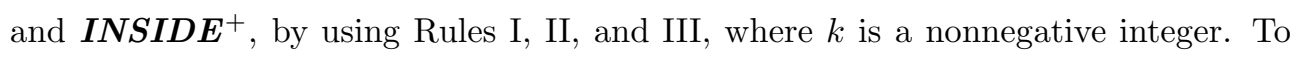
prove this claim, we need only to prove that for each $k, P_{k} \subseteq \boldsymbol{M A} \boldsymbol{X}(x)$, that is,

$$
P_{k} \subseteq M_{x 2} \cup M_{i n} M_{x 2} \cup M_{x 2} M_{c o} \cup M_{i n} M_{x 2} M_{c o} .
$$

Now let us use induction on the derivation length $k$ to prove it. $k=0$ :

$$
P_{0}=G_{x} \subseteq M_{x 2} .
$$

$k=1$ :

It is easy to see that

$$
\begin{aligned}
P_{1} & \subseteq G_{x}^{t} \cup G_{x} M_{o v} G_{x} \cup M_{i n} G_{x} \cup G_{x} M_{c o} \\
& \subseteq M_{x 2} \cup M_{i n} M_{x 2} \cup M_{x 2} M_{c o} .
\end{aligned}
$$

\section{Inductive Step:}


Assume $P_{k} \subseteq \boldsymbol{M A} \boldsymbol{X}(x)$, where $k \geq 1$. Then we wish to prove that $P_{k+1} \subseteq \boldsymbol{M A} \boldsymbol{X}(x)$.

Let $A x E \in P_{k+1}$, and let $A x E$ have a derivation with the derivation length $k+1$. We consider the following four cases (a)-(d).

Case (a) Use Rule I at the last step of its derivation. Then

$$
A x E:: A x C, C x E
$$

where both $A x C$ and $C x E$ are in $P_{k}$.

Hence, by the inductive assumption, both $A x C$ and $C x E$ are in $\boldsymbol{M A X}(x)$.

We consider the following 16 subcases. Let $M_{x 2}, M_{i n} M_{x 2}, M_{x 2} M_{c o}$, and $M_{i n}$ $M_{x 2} M_{c o}$ be called, respectively, the first, second, third, and fourth forms that the two relationships $A x C$ and $C x E$ each can take. Then, each subcase (ak) corresponds to the condition that $A x C$ and $C x E$ take the $i$ th and $j$ th forms, respectively, where $k=4(i-1)+j, 1 \leq i, j \leq 4$.

Subcase (al) If both $A x C$ and $C x E$ are in $M_{x 2}$, so is $A x E$.

Subcase (a2) If $A x C \in M_{x 2}$ and $C x E \in M_{i n} M_{x 2}$, then

$$
C x E:: C \text { insideD, DxE }
$$

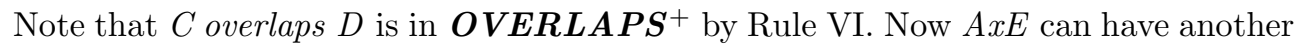
derivation that uses Rule II at the last step, namely,

$$
A x E:: A x C, C \text { overlaps } D, D x E
$$

Thus $A x E \in M_{x 2}$. This actually means that $A x E$ can be deduced by using only Rules I and II.

Subcase (a3) If $A x C \in M_{x 2}$ and $C x E \in M_{x 2} M_{c o}$, then

$$
C x E:: C x D, D \text { contains } E
$$

where $C x D \in M_{x 2}$ and $D$ contains $E \in C$ ONTAINS ${ }^{+}$.

Now $A x D$ can be derived from $A x C$ and $C x D$ by using Rule I. Thus, it is in $M_{x 2}$. Therefore, $A x E$ can have another derivation that uses Rule III(b) at the last step, namely,

$$
\text { AxE:: } A x D, D \text { contains } E
$$

This means $A x E \in M_{x 2} M_{c o}$.

Subcase (a4) If $A x C \in M_{x 2}$ and $C x E \in M_{i n} M_{x 2} M_{c o}$, then

$$
\begin{aligned}
& C x E \text { :: } C \text { insideD }, D x D^{\prime}, D^{\prime} \text { contains } E \\
& \text { where } \quad C \text { inside } D \in \boldsymbol{I N S I D E ^ { + }}, D x D^{\prime} \in M_{x 2} \\
& \text { and } D^{\prime} \text { containsE } \in \text { CONTAINS }{ }^{+} \text {. }
\end{aligned}
$$

By Rule III(a), we have

$$
C x D^{\prime}:: C \text { inside } D, D x D^{\prime}
$$


Hence, $C x D^{\prime} \in M_{i n} M_{x 2}$. By Rule I, we have

$$
A x D^{\prime}:: A x C, C x D^{\prime}
$$

Then, similar to Subcase (a2), we have $A x D^{\prime} \in M_{x 2}$. Furthermore, $A x E$ can be derived from $A x D^{\prime}$ and $D^{\prime}$ contains $E$ by using Rule III(b), namely,

$$
\text { AxE :: AxD', } D^{\prime} \text { contains } E \text {. }
$$

Thus $A x E \in M_{x 2} M_{c o}$.

Subcases (a5-a8) Each subcase (ai), where $5 \leq i \leq 8$, can be considered as the subcase $(\mathrm{a}(i-4))$ followed by using Rule III(a) at the last step. Thus $A x E$ is, respectively, in $M_{i n} M_{x 2}$ for Subcases (a5) and (a6), and in $M_{i n} M_{x 2} M_{c o}$ for Subcases (a7) and (a8).

Subcase (a9) If $A x C \in M_{x 2} M_{c o}$ and $C x E \in M_{x 2}$, then

$$
\begin{aligned}
& A x C:: A x B, B \text { contains } C \\
& \text { where } A x B \in M_{x 2} \text { and } B \text { contains } C \in M_{c o} .
\end{aligned}
$$

Note that $B$ overlaps $C$ can be derived from $C$ inside $B$ by using Rules VI and IV. Now $A x E$ can have another derivation that uses Rule II at the last step, namely,

$$
A x E:: \text { A } x, B \text { overlaps } C, C x E \text {. }
$$

Thus $A x E \in M_{x 2}$.

Subcase (a10) If $A x C \in M_{x 2} M_{c o}$ and $C x E \in M_{i n} M_{x 2}$, then (4) and (3) hold. Now we can derive $C$ overlaps $D$ by Rule VI, and $B$ overlaps $D$ from $C$ inside $B$ and $C$ overlaps $D$ by Rule VII. Moreover, $A x E$ can have another derivation that uses Rule II at the last step, namely,

$$
\text { AxE:: A } x B, B \text { overlaps } D, D x E \text {. }
$$

Thus $A x E \in M_{x 2}$.

Subcases (all-al2) Both subcases (all) and (al2) can be considered to be the subcases (a9) and (al0), respectively, followed by using Rule III(b) at the last step. Thus $A x E \in M_{x 2} M_{c o}$.

Subcases (al3-al6) Each subcase (ai), where $13 \leq i \leq 16$, can be considered as the subcase $(\mathrm{a}(i-4))$ followed by using Rule III(a) at the last step. Thus $A x E$ is, respectively, in $M_{i n} M_{x 2}$ for Subcases (al3) and (al4), and in $M_{i n} M_{x 2} M_{c o}$ for Subcases (al5) and (al6).

We summarize Case (a) in Table 1. If $A x C$ and $C x E$ take the forms in the positions $(i, 0)$ and $(0, j)$, respectively, where $1 \leq i, j \leq 4$, then $A x E$ has the form in the position $(i, j)$. 


\section{Case (b)}

Use Rule II at the last step of its derivation. Then

$$
\begin{aligned}
& A x E:: \text { AxE }, C \text { overlaps } C^{\prime}, C^{\prime} x E \\
& \text { where } \text { both } A x C \text { and } C^{\prime} x E \text { are in } P_{k} \\
& \text { and } \quad C \text { overlaps } C^{\prime} \in \boldsymbol{O} \boldsymbol{V E R \boldsymbol { R } \boldsymbol { A } \boldsymbol { P } \boldsymbol { S } ^ { + } .}
\end{aligned}
$$

Hence, by the inductive assumption, both $A x C$ and $C x E$ are in $\boldsymbol{M A X}(x)$.

Similar to Case (a), we also consider the following 16 subcases. Let $M_{x 2}, M_{i n} M_{x 2}$, $M_{x 2} M_{c o}$, and $M_{i n} M_{x 2} M_{c o}$ be called, respectively, the first, second, third, and fourth forms that the two relationships $A x C$ and $C^{\prime} x E$ each can take. Then, each subcase $(\mathrm{h} k)$ corresponds to the condition that $A x C$ and $C^{\prime} x E$ take the $i$ th and $j$ th forms, respectively, where $k=4(i-1)+j, 1 \leq i, j \leq 4$.

TABLE 1

Matrix Forms for the first Two Cases

\begin{tabular}{|l|l|l|l|l|}
\hline & $M_{x 2}$ & $M_{i n} M_{x 2}$ & $M_{x 2} M_{c o}$ & $M_{i n} M_{x 2} M_{c o}$ \\
\hline$M_{x 2}$ & $M_{x 2}$ & $M_{x 2}$ & $M_{x 2} M_{c o}$ & $M_{x 2} M_{c o}$ \\
\hline$M_{i n} M_{x 2}$ & $M_{i n} M_{x 2}$ & $M_{i n} M_{x 2}$ & $M_{i n} M_{x 2} M_{c o}$ & $M_{i n} M_{x 2} M_{c o}$ \\
\hline$M_{x 2} M_{c o}$ & $M_{x 2}$ & $M_{x 2}$ & $M_{x 2} M_{c o}$ & $M_{x 2} M_{c o}$ \\
\hline$M_{i n} M_{x 2} M_{c o}$ & $M_{i n} M_{x 2}$ & $M_{i n} M_{x 2}$ & $M_{i n} M_{x 2} M_{c o}$ & $M_{i n} M_{x 2} M_{c o}$ \\
\hline
\end{tabular}

Subcase (bl) If both $A x C$ and $C^{\prime} x E$ are in $M_{x 2}$, so is $A x E$.

Subcase (b2) If $A x C \in M_{x 2}$ and $C^{\prime} x E \in M_{i n} M_{x 2}$, then

$$
C^{\prime} x E:: C \text { inside } D, D x E
$$

$$
\text { where } C^{\prime} \text { inside } D \in \boldsymbol{I N S I D \boldsymbol { E } ^ { + }} \text { and } D x E \in M_{x 2} \text {. }
$$

We can derive $C^{\prime}$ overlaps $C$ from $C$ overlaps $C^{\prime}$ by using Rule IV, and $D$ overlaps $C$ from $C^{\prime}$ inside $D$ and $C^{\prime}$ overlaps $C$ by using Rule VII, and $C$ overlaps $D$ from $D$ overlaps $C$ by using Rule IV. Hence, $A x E$ can have another derivation that uses Rule II at the last step as follows:

$$
A x E:: A x C, C \text { overlaps } D, D x E \text {. }
$$

Thus $A x E \in M_{x 2}$.

Subcases (b3-b4) Both subcases (b3) and (b4) can be considered to be the subcases (bl) and (b2), respectively, followed by using Rule III(b) at the last step. Thus, $A x E \in M_{x 2} M_{c o}$ for these two subcases (b3) and (b4).

Subcases (b5-b8) Each subcase (bi), where $5 \leq i \leq 8$, can be considered as the subcase $(\mathrm{b}(i-4))$ followed by using Rule III(a) at the last step. Thus $A x E$ is, respectively, in $M_{i n} M_{x 2}$ for Subcases (b5) and (b6), and in $M_{i n} M_{x 2} M_{c o}$ for Subcases (b7) and (b8). 
Subcase (b9) If $A x C \in M_{x 2} M_{c o}$ and $C^{\prime} x E \in M_{x 2}$, then (4) holds. Now we can derive $B$ overlaps $C^{\prime}$ from $C$ inside $B$ and $C$ overlaps $C^{\prime}$ by using Rule VII. Then $A x E$ can have another derivation that uses Rule II at the last step as follows:

$$
A x E:: A x B, B \text { overlaps } C^{\prime}, C^{\prime} x E \text {. }
$$

Thus $A x E \in M_{x 2}$.

Subcase (bl0) If $A x C \in M_{x 2} M_{c o}$ and $C^{\prime} x E \in M_{i n} M_{x 2}$, then (4) and (5) hold. We can derive $B$ overlaps $C^{\prime}$ from $C$ inside $B$ and $C$ overlaps $C^{\prime}$ by Rule VII, then $C^{\prime}$ overlaps $B$ from $B$ overlaps $C^{\prime}$ by Rule IV, and $D$ overlaps $B$ from $C^{\prime}$ inside $D$ and $C^{\prime}$ overlaps $B$ by Rule VII, and $B$ overlaps $D$ from $D$ overlaps $D$ by Rule IV. So $A x E$ can have another derivation that uses Rule II at the last step as follows:

$$
\text { AxE :: A } x B, B \text { overlaps } D, D x E \text {. }
$$

Thus $A x E \in M_{x 2}$.

Subcases (bll-bl2) Both subcases (bll) and (bl2) can be considered to be the subcases (b9) and (bl0), respectively, followed by using Rule III(b) at the last step. Thus $A x E \in M_{x 2} M_{c o}$.

Subcases (bl3-bl6) Each subcase (bi), where $13 \leq i \leq 16$, can be considered as the subcase $(\mathrm{b}(i-4))$ followed by using Rule III(a) at the last step. Thus $A x E$ is, respectively, in $M_{i n} M_{x 2}$ for Subcases (bl3) and (bl4), and in $M_{i n} M_{x 2} M_{c o}$ for Subcases (bl5) and (bl6).

Therefore, in Case (b), we have the same summary table (Table 1) for the forms of $A x E$ as in Case (a). If $A x C$ and $C^{\prime} x E$ take the forms in the positions $(i, 0)$ and $(0, j)$, respectively, where $1 \leq i, j \leq 4$, then $A x E$ has the form in the position $(i, j)$.

Case (c)

Use Rule III(a) at the last step of its derivation. Then

$$
\begin{aligned}
& A x E:: \text { A inside } C, C x E \\
& \text { where } \quad A \text { inside } C \in \boldsymbol{I N S I D \boldsymbol { E } ^ { + }} \text { and } C x E \in P_{k} .
\end{aligned}
$$

Hence, by the inductive assumption, $C x E$ are in $\boldsymbol{M A X}(x)$.

We consider the following four subcases.

Subcase (cl) If $C x E \in M_{x 2}$, then $A x E \in M_{i n} M_{x 2}$.

Subcase (c2) If $C x E \in M_{i n} M_{x 2}$, then (3) holds. Now $A$ inside $D$ can be deduced in one step from $A$ inside $C$ and $C$ inside $D$ by using Rule I. Furthermore, $A x E$ can have another derivation that uses Rule III(a) at the last step as follows:

$$
A x E:: \text { A inside } D, D x E
$$

Thus $A x E \in M_{i n} M_{x 2}$.

Subcase (c3) If $C x E \in M_{x 2} M_{c o}$, this case can be considered to be Subcase (cl), followed by using Rule III(b) at the last step. Thus $A x E \in M_{i n} M_{x 2} M_{c o}$.

Subcase (c4) If $C x E \in M_{i n} M_{x 2} M_{c o}$, this case can be considered to be Subcase (c2), followed by using Rule III(b) at the last step. Thus $A x E \in M_{i n} M_{x 2} M_{c o}$. 


\section{Case (d)}

Use Rule III(b) at the last step of its derivation. Then

$$
A x E:: A x C, C \text { contains } E
$$

where $\quad A x C \in P_{k}$ and $C$ contains $E \in$ CONTAINS ${ }^{+}$.

Hence, by the inductive assumption, $A x C$ are in $\boldsymbol{M A} \boldsymbol{X}(x)$.

Similar to Case (c), we also consider the following four subcases.

Subcase (dl) If $A x C \in M_{x 2}$, then $A x E \in M_{x 2} M_{c o}$.

Subcase (d2) If $A x C \in M_{i n} M_{x 2}$, then $A x E \in M_{i n} M_{x 2} M_{c o}$.

Subcase (d3) If $A x C \in M_{x 2} M_{c o}$, then (4) holds. Now $B$ contains $E$ can be deducible from $B$ contains $C$ and $C$ contains $E$. Furthermore, $A x E$ can have another derivation that uses Rule III(b) at the last step as follows:

$$
\text { AxE:: } A x B, B \text { contains } E \text {. }
$$

Thus $A x E \in M_{x 2} M_{c o}$.

Subcase (d4) If $A x C \in M_{i n} M_{x 2} M_{c o}$, this case can be considered to be Subcase (d3), followed by using Rule III(a) at the last step. Thus $A x E \in M_{i n} M_{x 2} M_{c o}$. This completes the proof of Claim 3.2.

\section{REFERENCES}

[1] A. V. Aho, M.R. Garey, and J. D. Ullman, The Transitive Reduction of a Directed Graph, SIAM J. of Comput, 1:2(1972), pp. 131-137.

[2] A. V. Aho, J. E. Hopcroft, and J. D. Ullman, The Design and Analysis of Computer Algorithms, Addison-Wesley, Reading. Massachusetts, 1974.

[3] A. V. Aho, J. E. Hopcroft, And J. D. Ullman, Data Structures and Algorithms, AddisonWesley, Reading, Massachusetts, 1983.

[4] T. Arndt and S.-K. Chang, Image Sequence Compression by Iconic Indexing, in: Proc. IEEE Workshop Visual Languages, Rome, Italy, 177-182, 1989.

[5] S. BAASe, Computer Algorithms: Introduction to Design and Analysis, second edition, Addison-Wesley, Reading, Massachusetts, 1988.

[6] C. C. Chang and S. Y. Lee, Retrieval of Similar Pictures on Pictorial Databases, Pattern Recognition, 24:7(1991), pp. 675-680.

[7] S.-K. Chang, Pictorial Information Systems, Prentice-Hall, Englewood Cliff's, N.J., 1989.

[8] S.-K. Chang AND A. Hsu, Image Information Systems: Where do we go from here?, IEEE Transactions on Knowledge and Data Engineering, 4:5(1992), pp. 431-442.

[9] S.-K. Chang, E. Jungert, And Y. Li, Representation and Retrieval of Symbolic Pictures Using Generalized 2D Strings, in: SPIE Proc. Visual Commun. and Image Processing, Philadelphia, Nov. 5-10, 1989.

[10] S.-K. Chang, E. Jungert, And Y. Li, The Design of Pictorial Databases Based upon the Theory of Symbolic Projections, in: Proc. Int. Symposium on Large Spatial Databases (SSD), Springer-Verlag, 1989.

[11] S.-K. Chang and T. Kunit, Pictorial Database Systems, IEEE Comput. Mag., Special Issue on Pictorial Information Systems, 14(1981), pp. 13-21. 
[12] S.-K. Chang And Y. LI, Representation of Multi-resolution Symbolic and Binary Pictures Using 2DH Strings, in: Proc. IEEE Workshop Languages for Automation, 190-195, 1988.

[13] S.-K. Chang, Q. Y. ShI, And C. W. YAN, Iconic Indexing by 2-D Strings, IEEE Trans. Pattern Anal Machine Intell, 9:3(1987), pp. 413-428.

[14] S.-K. Chang, C. W. Yan, D. C. Dimitroff, and T. Arndt, An Intelligent Image Database System, IEEE Trans. Software Engineering, 14:5(1988), pp. 681-688.

[15] G. Costagliola, F. Ferrucci, G. Tortora, and M. Tucci, Non-Redundant 2D Strings, IEEE Transactions on Knowledge and Data Engineering, 7:2(1995), pp. 347-350.

[16] G. Costagliola, G. Tortora, and T. Arndt, A Unifying Approach to Iconic Indexing for $2 D$ and 3D Scenes, IEEE Transactions on Knowledge and Data Engineering, 4:3(1992), pp. 205-222.

[17] G. Costagliola, M. Tucci, and S.-K. Chang, Representing and Retrieving Symbolic Pictures by Spatial Relations, in: Visual Database Systems, North-Holland, 49-59, 1992.

[18] M. J. Fischer And A.R. Meyer, Boolean Matrix Multiplication and Transitive Closure, Proceedings of the Twelfth Annual Symposium on Switching and Automation Theory, East Lansing, Michigan, 129-131, 1971.

[19] M. Flickner, H. Sawhney, and W. Niblack, et Al., Query by Image and Video Content: The QBIC System, IEEE Computer, 23-32, September 1995.

[20] M. E. Furman, Application of a Method of Fast Multiplication of Matrices in the Problem of Finding the Transitive Closure of a Graph, Dokl. Akad. Nauk SSSR, 11:5(1970), pp. 1252.

[21] V. N. Gudivada And V. V. Raghavan, Design and Evaluation of Algorithms for Image Retrieval by Spatial Similarity, ACM Transactions on Information Systems, 13:2(1995), pp. $115-144$.

[22] T.-Y. Hou, P. Lui, And M. Y. Chui, A Content-based Indexing Technique Using Relative Geometry Features, Proceedings of SPIE, Image Storage and Retrieval Systems, the International Society for Optical Engineering, Vol. 1662, 1992.

[23] C. L. Jackins and S. L. Tanimoto, Oct-trees and Their Use in Representing Threedimensional Objects, Comput. Graphics and Image Processing, 14:3(1980), pp. 249-270.

[24] E. Jungert, Extended Symbolic Projection Used in a Knowledge Structure for Spatial Reasoning, in: Proc. 4th BPRA Conf. Pattern Recognition, Springer-Verlag, Cambridge, March 28-30, 1988.

[25] E. Jungert And S.-K. Chang, An Algebra for Symbolic Image Manipulation and Transformation, in: Visual Database Systems, North-Holland, 301-317, 1989.

[26] S.-Y. Lee, M.-K. Shan, And W.-P. Yang, Similarity Retrieval of Iconic Image Database, Pattern Recognition, 22:6(1989), pp. 675-682.

[27] D. J. Meagher, Geometric Modeling Using Octree Encoding, Comput. Graph, and Image Processing, 19:2(1981), pp. 129-147.

[28] D. M. Moyles and G. L. Thompson, Finding a Minimum Equivalent Graph of a Digraph, J. ACM, 16(1969), pp. 455-460.

[29] A. Pentland, R. W. Picard, and S. Sclroff, Photobook: Content-based Manipulation of Image Databases, Proceedings of SPIE - Storage and Retrieval for Image and Video Database II, (SPIE, Bellingham, WA), San Jose, CA, 34-47, Feb. 6-10, 1994.

[30] A. P. Sistla, C. Yu, And R. Haddad, Reasoning about Spatial Relationships in Picture Retrieval Systems, Proceedings of the 20th International Conference on Very Large Databases, Santiago, Chile, 570-581, 1994.

[31] H. Tamura and N. Yokoya, Image Database Systems: A Survey, Pattern Recognition, Vol. 17, 29-43, 1984.

[32] S. L. Tanimoto, An Iconic Symbolic Data Structuring Scheme, Pattern Recogn. and Artificial Intell, Academic Press, New York, 452-471, 1976. 
[33] G. Tortora, G. Costagliola, T. Arndt, and S.-K. Chang, Pyramidal Algorithms for Iconic Indexing, Computer Vision, Graphics, and Image Processing, 53(1990), pp. 26-56.

[34] Q.-L. Zhang, A Unified Framework for Iconic Indexing of Spatial Relationships in Image Databases, Ph.D. Thesis, Department of Mathematics, Statistics, and Computer Science, University of Illinois, Chicago, 1996.

[35] Q.-L. Zhang, S.-K. Chang, and S. S.-T. Yau, A Unified Approach to Indexing Images in Image Databases, Proceedings of the First International Workshop on Image Databases and Multi-Media Search, Amsterdam, The Netherlands, 99-106, August 22-23, 1996.

[36] Q.-L. Zhang, S.-K. Chang, and S. S.-T. Yau, A Unified Approach to Iconic Indexing, Retrieval, and Maintenance of Spatial Relationships in Image Databases, Journal of Visual Communication and Image Representation, Special Issue on Indexing, Storage, Retrieval and Browsing of Images and Video, Academic Press, 7:4(1996), pp. 307-324.

[37] Q.-L. Zhang, S.-K. Chang, and S. S.-T. Yau, An Experimental Result in Image Indexing using GEP-2D Strings, Image Databases and Multimedia Search (A. Smeulders and R. Jain, eds.), World Scientific Pub. Co., 127-146, 1997.

[38] Q.-L. Zhang, S.-K. Chang, And S. S.-T. YaU, On Consistency Checking of Spatial Relationships in Content-based Image Database Systems, Preprint, 2000.

[39] Q.-L. Zhang, S.-K. Chang, and S. S.-T. Yau, The Consistency Problem on Content-based Pictorial Description in Pictorial Database Systems, Communications in Information and Systems, International Press, 1:2(2001), pp. 225-240.

[40] Q.-L. Zhang and S. S.-T. Yau, Finding Minimal and Maximal Sets of Spatial Relationships in Picture Retrieval Systems, Department of Mathematics, Statistics, and Computer Science, University of Illinois, Chicago, Preprint, 1995.

[41] Q.-L. Zhang And S. S.-T. Yau, A New Iconic Indexing for $2 D$ and $3 D$ Scenes, Proceedings of the 2nd Chinese World Congress on Intelligent Control and Intelligent Automation (CWCICIA 97), Xi'an, P. R. China, 1667-1672, June 23-27, 1997.

[42] Q.-L. Zhang and S. S.-T. YaU, A General Approach to Indexing and Retrieval of Images in Image Databases, Communications in Information and Systems, International Press, 3:1(2003), pp. 61-73.

[43] Q.-L. Zhang and S. S.-T. Yau, A Stepwise Approximation of Intractable Spatial Constraints in Image Queries, Communications in Information and Systems, International Press, 3:3(2003), pp. 203-221.

[44] Q.-L. Zhang And S. S.-T. Yau, On Intractability of Spatial Relationships in Content-based Image Database Systems, Communications in Information and Systems, International Press, 4:2(2004), pp. 181-190. 